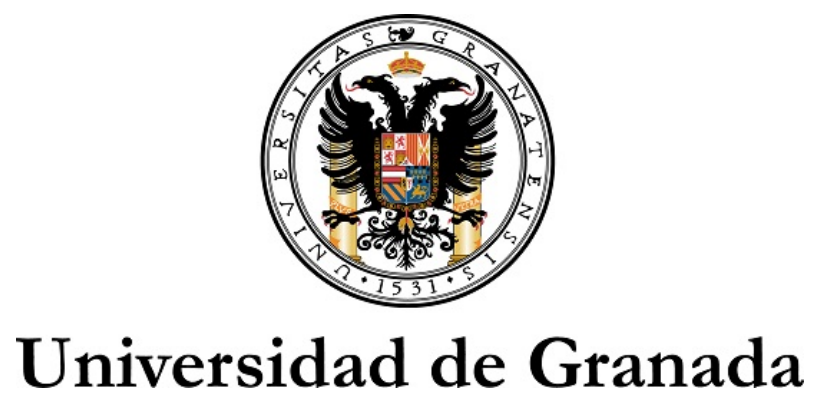

Máster en Física: Radiaciones, Nanotecnología, Partículas y Astrofísica

\author{
TRABAJO DE FIN DE MÁSTER
}

\title{
Estudio de las propiedades de los fotomultiplicadores de silicio (SiPM)
}

por

Alejandro Sánchez López

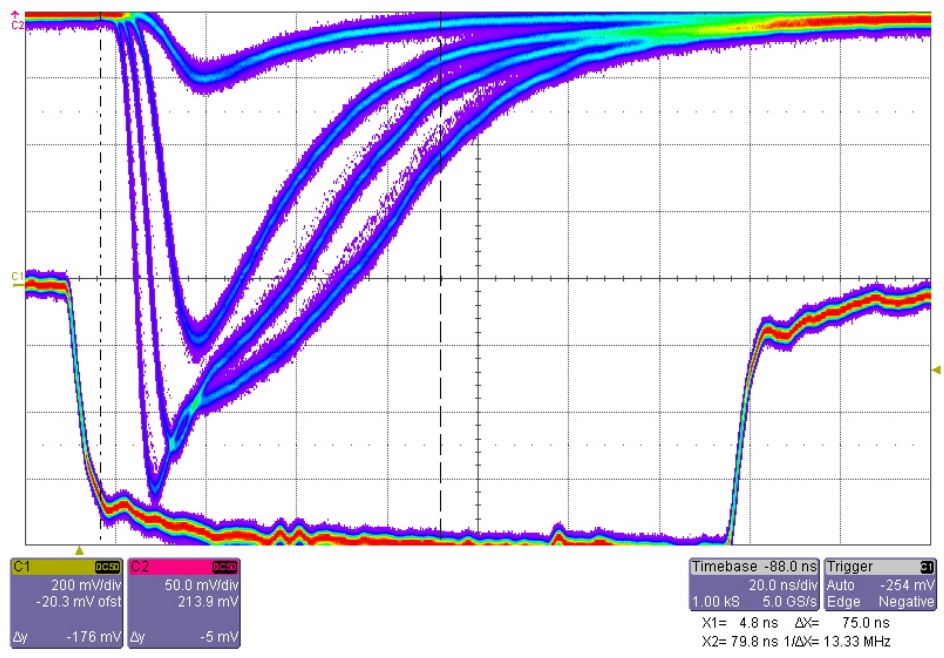





\title{
Estudio de las propiedades de los fotomultiplicadores de silicio $(\mathrm{SiPM})$
}

\author{
Alejandro Sánchez López \\ 23 de junio de 2015
}

Facultad de Ciencias de la Universidad de Granada. Departamento de Física Teórica y del Cosmos.

Tutor: Sergio Navas Concha

Firma:

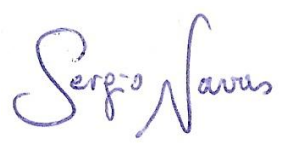





\section{Índice}

$\begin{array}{lll}\text { I Introducción } & 3\end{array}$

1. ¿Qué es un SiPM? $\quad 4$

1.1. Reflexión en la superficie . . . . . . . . . . . . . . . 5

1.1.1. Absorción en el silicio. . . . . . . . . . . . . . . 6

1.2. Eficiencia cuántica . . . . . . . . . . . . . . . 7

1.3. Factor de llenado geométrico . . . . . . . . . . . . . . . 7

1.4. Eficiencia de detección de fotones . . . . . . . . . . . . . . . . 9

1.5. Parámetros intrínsecos de operación . . . . . . . . . . . . . . . . 9

1.5.1. Breakdown Voltage y Over-voltage............... 9

1.5.2. Modo Geiger y ganancia del SiPM . . . . . . . . . . . . . 10

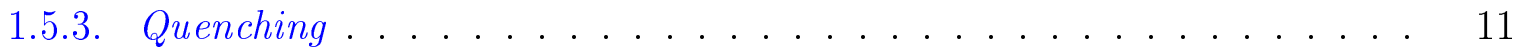

1.5.4. Tiempo de recuperación . . . . . . . . . . . . . . 11

1.6. Fuentes de ruido en el SiPM . . . . . . . . . . . . . . . 12

1.6.1. Cuentas oscuras (Dark Counts) . . . . . . . . . . 12

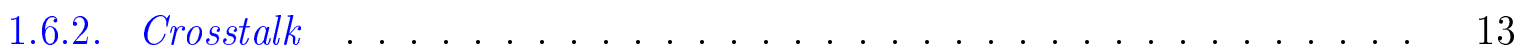

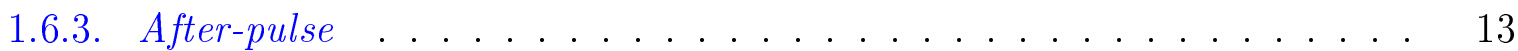

1.7. Aplicaciones presentes y futuras de los fotomultiplicadores de silicio . . . . . . 14

II Experimento. Descripción y métodos. 17

2. Metodología para la determinación de la ganancia $r$

3. Metodología para la determinación de las cuentas oscuras y el Crosstalk 23

III Resultados para la Ganancia 25

IV Resultados para cuentas oscuras y Crosstalk 33

4. Comportamiento de las cuentas oscuras 33

5. Estimación y resultados de la probabilidad de Crosstalk 37 
V Conclusiones

A. Descripción de los módulos electrónicos. 


\section{Resumen}

A lo largo de este Trabajo de Fin de Máster, se pretende introducir el concepto de fotomultiplicador de silicio (SiPM), explicar cómo funciona este tipo de detector de estado sólido, explorar sus parámetros de operación más importantes y trabajar con él en el laboratorio para aportar un conocimiento teórico y experimental que ayude a comprender su funcionamiento y potencial utilidad en experimentos presentes y futuros. Los propósitos principales de este trabajo son, por tanto, caracterizar adecuadamente el SiPM que se encuentra en el laboratorio especializado de Física de Partículas del Departamento de Física Teórica y del Cosmos de la Universidad de Granada, conociendo bien su fundamento teórico y dedicar una buena parte del tiempo a trabajar en el laboratorio en la medida experimental de las magnitudes fundamentales como son los voltajes de operación, la ganancia y las fuentes de ruido, así como la dependencia de las mismas con otras variables

como la temperatura. No sólo esto último aporta una primera y detallada experiencia en un laboratorio de Física de Partículas, imprescindible a modo de base para los próximos años y con uno de los detectores de mayor proyección en la próxima década en muchos campos de la ciencia, sino que es el complemento perfecto a unos estudios de Licenciatura y a un Máster eminentemente teóricos, en los que son pocas las opciones de trabajar de verdad en un laboratorio en el que aprender a desenvolverse.

Palabras clave: Fotomultiplicador de silicio, fotodetectores, fotodiodo de avalancha Geiger, detector de fotones. 



\section{Parte I}

\section{Introducción}

Los fotodiodos semiconductores se desarrollaron a principios de los años cuarenta, al tiempo que se comercializaban los tubos fotomultiplicadores (PMT) [1]. Sólo en los años recientes, con la invención de los fotodiodos de avalancha en modo Geiger, se ha conseguido que estos fotodetectores alcancen la sensibilidad de los PMT's. La evolución comenzó en los años sesenta con los fotodiodos PIN (P-I-N o semiconductores dopados con impurezas donadoras, acoplados a un aislante y a éste a su vez a un semiconductor dopado con impurezas aceptadoras), un dispositivo muy exitoso que se utiliza todavía hoy día en los detectores de física de altas energías y en otra gran variedad de disciplinas. El siguiente paso fue el desarrollo de los fotodiodos de avalancha (APD) con amplificadores de alta ganancia dando lugar a una considerable disminución del ruido pero sin alcanzar todavía el nivel de detección de un único fotón [2].

Por su parte, el fotomultiplicador de Silicio (SiPM en adelante), desarrollado a finales del pasado milenio, acomete finalmente con éxito el reto de detectar, medir temporalmente y cuantificar señales poco luminosas hasta el nivel de un solo fotón. Los SiPM ofrecen una alternativa altamente atractiva que replica minuciosamente las propiedades de detección en condiciones de baja luminosidad de los PMT, proporcionando al mismo tiempo todos y cada uno de los beneficios de los detectores de estado sólido. Un SiPM ofrece la opción de trabajar con bajos voltajes de operación (menos de $100 \mathrm{~V}$ normalmente), posee insensibilidad a los campos magnéticos, destaca por su robustez mecánica y excelente uniformidad de la respuesta y es además muy compacto en dimensiones. Debido a estas características, el SiPM ha ganado rápidamente importancia por su rendimiento en los campos de procesado de imágenes médicas, biofotónica, física de altas energías, astrofísica, LiDAR ${ }^{1}$, etc.

En este Trabajo de Fin de Máster se ha realizado una búsqueda y análisis de la bibliografía especializada en el campo de los fotodiodos de avalancha en general, y de los SiPM en particular. Comprender en profundidad su estructura interna y los parámetros de operación fundamentales ha sido el punto de partida, el cual se desarrolla en la sección 1. Con las bases teóricas adquiridas, hemos ido al laboratorio especializado de la Universidad de Granada a realizar medidas de caracterización del fotomultiplicador de silicio, hallando su ganancia, valores fundamentales del voltaje, abordando la siempre presente cuestión del ruido intrínseco del detector y electrónico, para caracterizarlo adecuadamente y poder tenerlo en cuenta en

\footnotetext{
${ }^{1}$ Light Detection and Ranging o Laser Imaging Detection and Ranging es una tecnología que permite determinar la distancia desde un emisor láser a un objeto o superficie utilizando un haz láser pulsado. Utilizado, por ejemplo, en experimentos de física de rayos cósmicos como en el Observatorio Pierre Auger.
} 
futuros experimentos a realizar con el SiPM del laboratorio. Además, se ha realizado un estudio de la evolución de todas las magnitudes fundamentales con la temperatura. Para obtener las medidas, ha sido necesario adquirir una metodología disciplinada en el laboratorio y un importante nivel de comprensión y manejo de la electrónica del mismo, por lo que se dedica la parte II a una detallada explicación de los procedimientos que se han seguido en el laboratorio para preparar, verificar y tomar finalmente cada uno de los datos. Los resultados que se han obtenido a través del análisis exhaustivo utilizando el paquete ROOT [3] para la ganancia, valores del voltaje y ruido, se exponen y son discutidos en profundidad en las partes III y IV. Finalmente, la parte $\mathrm{V}$ se dedica a resumir las principales conclusiones obtenidas de los análisis. Tras las referencias bibliográficas se incluye un Apéndice con una breve descripción de los módulos electrónicos que forman parte del experimento.

\section{1. ¿Qué es un SiPM?}

Los Fotomultiplicadores de Silicio (figura 1) son nuevos detectores de estado sólido de fotones basados en las propiedades de los fotodiodos semiconductores (uniones PN en su nivel más básico) y cuyo potencial es hoy día objeto de intenso estudio en muchos grupos que buscan aumentar sus aplicaciones a diversos campos como los previamente mencionados. Un SiPM consta de una matriz de, típicamente, entre $1 \times 1$ y $5 \times 5 \mathrm{~mm}^{2}$ y del orden de 1000 microceldas independientes (píxeles) por cada $\mathrm{mm}^{2}$ conectadas en paralelo. Cada píxel está formado por un fotodiodo y una resistencia de extinción o quenching en serie. El fotodiodo trabaja unos voltios por encima de su Breakdown Voltage, esto es, el voltaje de alimentación para el cual se produce una cascada eléctrica siempre que se genere un fotoelectrón en el volumen activo (modo de operación Geiger). Para ser sensible a sucesivos fotones, cada avalancha es interrumpida mediante las resistencias.

Una característica fundamental de este modo de operación Geiger con el que se trabaja en los SiPM, es que la carga que da lugar a una avalancha puede ser producida por un proceso de absorción de un fotón, por una excitación térmica (ruido térmico), o puede ser emitida por un defecto en la propia red cristalina del silicio (after-pulse). Más adelante en este trabajo analizaremos las implicaciones de estas características en las tomas de datos. 


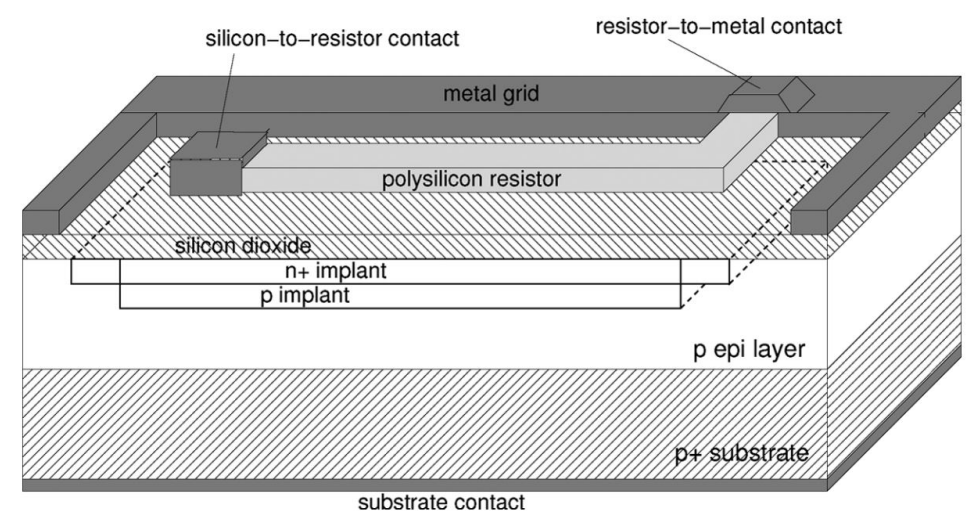

Figura 1: Esquema de la estructura interna de cada una de las microcélulas de un SiPM estándar [2].

Durante la cascada eléctrica, estos portadores de carga primarios (fotoelectrones) aumentan su número considerablemente debido al proceso de avalancha de forma que se consigue alcanzar altos valores de la Ganancia $\left(G \sim 10^{6}\right)$ comparables a los que proporciona un PMT convencional, pero con una mucho mejor resolución debido a que la estructura en píxeles puede fabricarse con una alta uniformidad, por lo que el número de electrones resultantes de cada proceso de cascada eléctrica es aproximadamente constante, sin importar qué píxel es el que ha dado señal de lectura.

En lo sucesivo, se comentan con brevedad los parámetros más relevantes que permiten conocer y caracterizar la naturaleza de este detector.

\subsection{Reflexión en la superficie}

Cuando los fotones que proceden de un medio de bajo índice de refracción, como el aire, inciden sobre una superficie de alto índice de refracción, como el silicio $(n=3,5)$, una gran cantidad de fotones son reflejados. Además, normalmente un fotodiodo de silicio está cubierto de una fina capa de óxido de silicio $(n=1,46)$ de modo que esta capa también influye al reflejarse fotones en la interfase óxido-SiPM.

Dependiendo de la longitud de onda y del espesor del óxido de silicio, en torno a un 20-30\% de la luz incidente puede perderse por simple reflexión e incluso pueden darse fenómenos de interferencia. Esto produce una seria reducción de la llamada Eficiencia Cuántica (QE) del fotodiodo, que se definirá a continuación. Afortunadamente, las pérdidas por reflexión puede paliarse significativamente a un máximo de un $10 \%$ utilizando recubrimientos antirreflejantes hechos de varias capas muy finas con diferentes índices de refracción. 


\subsubsection{Absorción en el silicio.}

Una vez que los fotones han conseguido atravesar las capas de recubrimiento y llegan al seno del SiPM, son absorbidos casi en su totalidad. La longitud de absorción, inversa del coeficiente de absorción $\alpha$, depende fuertemente de la longitud de onda $\lambda$ de la luz incidente, tal y como puede verse en la figura 2, siendo el flujo de fotones a una distancia $d$ de la superficie, $I(d)$ :

$$
I(d)=I_{0} \exp (-\alpha d)
$$

donde $I_{0}$ es el flujo incidente que entra al silicio.

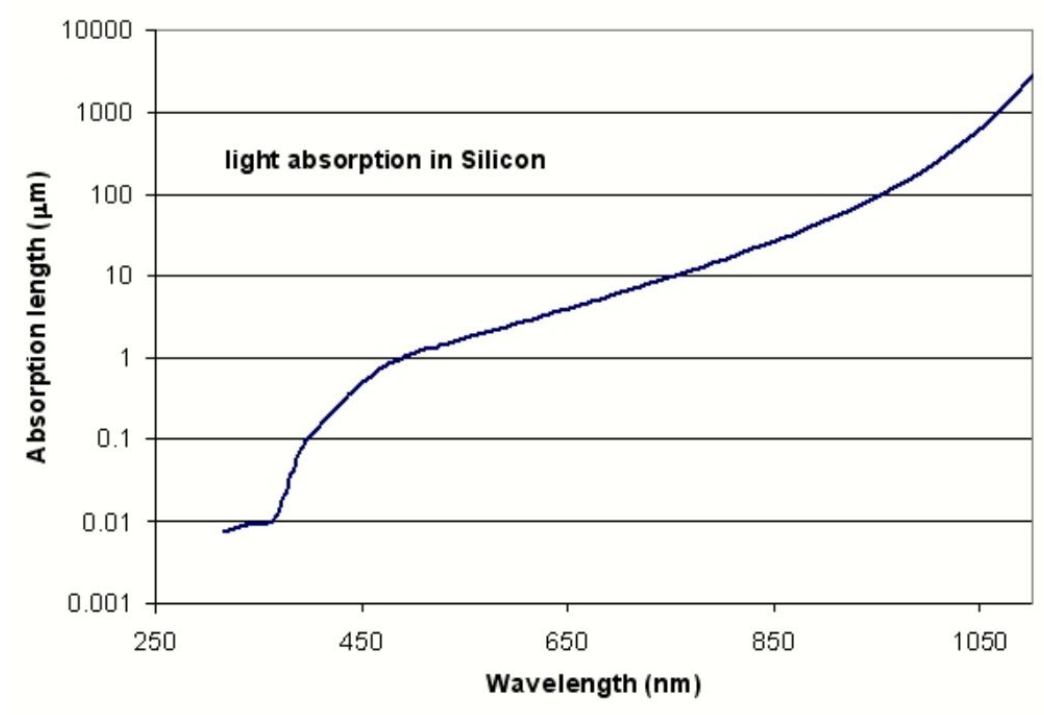

Figura 2: Longitud de absorción de los fotones en función de la longitud de onda para el silicio. $[3]$

A energías por debajo del gap entre las bandas (bandgap), 1,12 eV, los fotones sólo pueden ser absorbidos por electrones libres y el coeficiente de absorción se vuelve demasiado pequeño. Sin embargo, por encima del bandgap, los fotones pueden ser absorbidos por electrones de la banda de valencia que "saltan" hacia la banda de conducción. En este proceso se requiere adicionalmente un fonón en aras de la conservación del momento debido a la estructura de bandas indirecta del silicio, es decir, los momentos de los electrones y huecos en las bandas de valencia y de conducción no son iguales, de modo que los fotones no pueden absorberse o emitirse directamente, sino que el electrón debe pasar por un estado intermedio en el que transfiere o recibe momento mediante fonones de alto momento ${ }^{2}$ propios de la red cristalina. Las probabilidades de que este proceso ocurra son pequeñas, de modo que la probabilidad de

\footnotetext{
${ }^{2}$ Debido a que estamos por encima del bandgap, pero no demasiado.
} 
absorción se vuelve pequeña, motivo por el cual el SiPM se vuelve tan eficiente en la detección.

Para energías del fotón superiores, los fonones requeridos para la absorción son cada vez de menor energía, de modo que se vuelve mucho más probable que el proceso de absorción ocurra ( $\alpha$ aumenta considerablemente). Por encima de los 3,4 eV $(\lambda \sim 350 \mathrm{~nm})$ la transición directa entre bandas se vuelve posible y se dispara el valor de $\alpha$.

Por otra parte, la capa superior del fotodiodo disminuye la sensibilidad a las longitudes de onda cortas. Esto se debe a que esta capa suele tener una alta concentración de dopantes para asegurar una resistencia en serie pequeña y una respuesta uniforme. Además, esta capa $p^{++}$ (o $n^{++}$) no puede ser mermada, y los electrones y huecos creados por la absorción de un fotón en esta capa tienen una vida media muy corta debido a que se recombinan enseguida o deben moverse por difusión y pueden recombinarse en los estados de la interfase en la superficie. Por este motivo, la sensibilidad de los fotodiodos a los fotones de $\lambda$ corta, es decir, de pequeña longitud de absorción, es así ampliamente reducida.

Por su parte, los fotones de $\lambda$ larga poseen muy pequeños $\alpha$ y pueden, por tanto, atravesar todo el detector sin ser absorbidos ni detectados. Véase [3] para una amplia discusión de los efectos de difusión en las redes cristalinas de los semiconductores.

\subsection{Eficiencia cuántica}

Cuando los fotones llegan a un fotodiodo como es el caso de nuestro SiPM, se crean pares electrón-hueco si la energía de la luz incidente es superior al gap de energía, como se ha mencionado anteriormente. El ratio entre el número de pares electrón-hueco generados y el número total de fotones incidentes es lo que se conoce como eficiencia cuántica (QE, por las siglas en inglés de "Quantum Efficiency"), expresada comúnmente como un porcentaje ( \%), que depende de la longitud de onda de la luz incidente, como puede verse en la figura 3, alcanzándose valores de la $\mathrm{QE}$ de en torno al $80 \%$ para $\lambda \approx 450 \mathrm{~nm}$. Como veremos posteriormente, la tarea de nuestro SiPM es ahora amplificar la señal, generando una cascada de portadores de carga a partir de la creación del primer fotoelectrón.

\subsection{Factor de llenado geométrico}

El factor de llenado del SiPM es el ratio entre el área del dispositivo que es sensible a la detección de fotones (células o píxeles de sillicio) y el área total del mismo. El detector posee una cuadrícula que engloba a todos los píxeles la cual no es sensible a los fotones incidentes, por lo que una parte de la información se pierde mediante esta vía. Generalmente, cuanto más pequeño sea el tamaño del píxel, menor será el factor de llenado. Véase la figura 4 para ilustrar 
esta característica y la tabla 1 para ver las variaciones del factor de llenado con distintos parámetros.

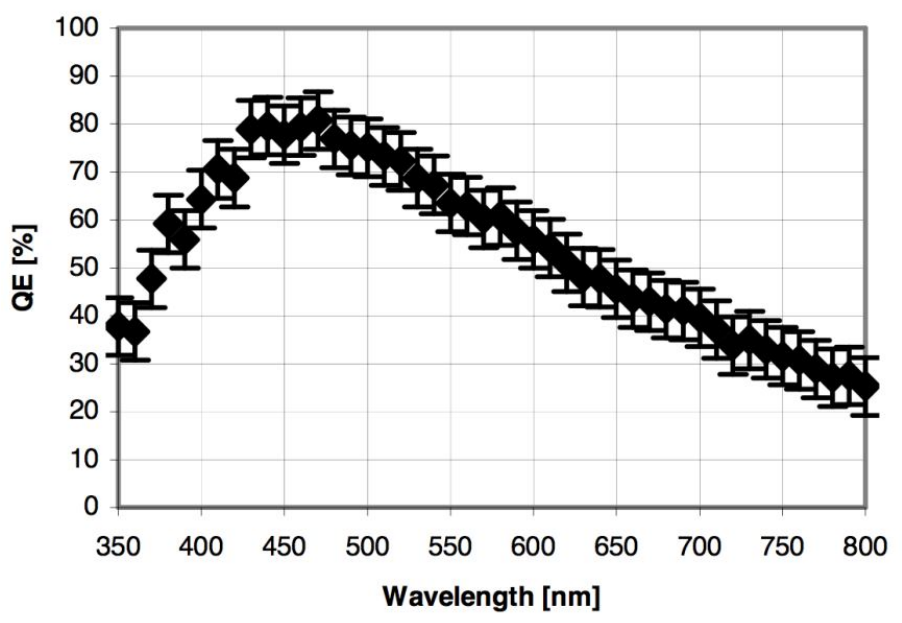

Figura 3: Eficiencia cuántica del área activa en función de la longitud de onda para el modelo de SiPM Hamamatsu 0-50-2 con $400 \frac{\text { celdas }}{\mathrm{mm}^{2}}$. Las barras de error denotan el error sistemático de medida [4]

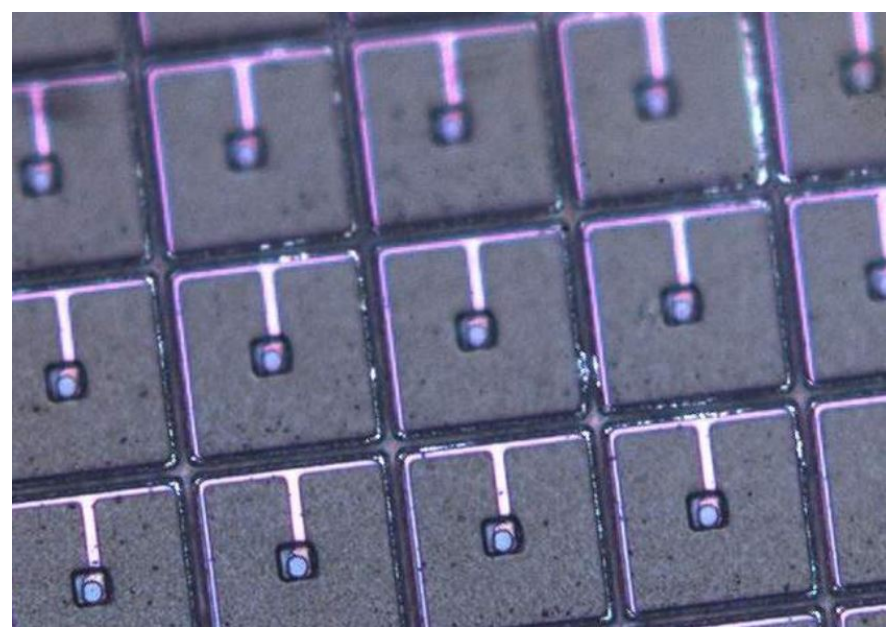

Figura 4: Imagen magnificada de un fotodiodo de avalancha que trabaja en modo Geiger (GAPD) [4]. En ella, apreciamos claramente el tamaño de los píxeles o células sensibles a recibir fotones y la cuadrícula que los engloba y que no forma parte del detector sensible, de manera que la información que incide en ésta última se pierde. Cada píxel tiene unas dimensiones de $43 \times 43 \mathrm{~mm}^{2}$. 


\begin{tabular}{|c|c|c|}
\hline \multicolumn{3}{|c|}{ Para un SiPM de $3 \times 3 \mathrm{~mm}^{2}$} \\
\hline \hline Número de píxeles & 3600 & 900 \\
\hline Tamaño del píxel $\left(\mu \mathrm{m}^{2}\right)$ & $50 \times 50$ & $100 \times 100$ \\
\hline Factor de llenado & $61,5 \%$ & $78,5 \%$ \\
\hline
\end{tabular}

Cuadro 1: Variación del Factor de llenado para distintos diseños de un SiPM de $3 \times 3 \mathrm{~mm}^{2}$.

\subsection{Eficiencia de detección de fotones}

Un parámetro muy importante de un fotodetector es la eficiencia con la que los fotones de una cierta longitud de onda pueden ser detectados (PDE, por las siglas en inglés de "Photon Detection Efficiency"). Para un SiPM, la PDE puede ser factorizada como producto de tres cantidades

$$
P D E=\epsilon_{g e o} \cdot Q E \cdot \epsilon_{\text {trigger }}
$$

donde $\epsilon_{\text {geo }}$ denota el factor de llenado geométrico, QE es la eficiencia cuántica y $\epsilon_{\text {trigger }}$ es la probabilidad combinada de que los electrones y huecos inicien una cascada eléctrica (modo Geiger).

\subsection{Parámetros intrínsecos de operación}

Supongamos que un fotón incidente llega al núcleo activo del píxel de silicio y genera un fotoelectrón. Analicemos las características fundamentales de los sucesos que ocurren en el detector para que podamos detectar la señal que corresponde a la interacción de dicho único fotón.

\subsubsection{Breakdown Voltage y Over-voltage}

Como es sabido, un diodo básico necesita ser sometido a una diferencia de potencial externa, que llamaremos en este trabajo $V_{\text {bias }}$, de manera que en su interior se genere un campo eléctrico asociado que acelere a los portadores libres, es decir, se induce un movimiento de cargas cuyo sentido depende de la carga eléctrica del portador ${ }^{3}$. Si el $V_{\text {bias }}$ al que sometemos el SiPM es demasiado bajo, el campo eléctrico no será suficientemente intenso como para acelerar al fotoeléctrón y éste tendrá una probabilidad alta de recombinarse, desbaratándose las opciones de detectar la luz incidente. Si continuamos aumentando el $V_{\text {bias }}$, poco a poco llegaremos a valores del campo eléctrico interno del fotodiodo con los que ya seremos capaces de acelerar el fotoelectrón y, mediante mecanismos que trataremos en los puntos siguientes, conseguiremos

\footnotetext{
${ }^{3}$ Obviamente, en este trabajo se asume que los "huecos" o ausencia de electrón en un determinado estado, poseen una carga eléctrica positiva (ausencia de una carga negativa).
} 
aumentar considerablemente el número de electrones arrancados y acelerados en el diodo, cuya carga podrá ser detectada y medida. En base a ello, se define el Breakdown Voltage ( $\left.V_{b r}\right)$ como el $V_{\text {bias }}$ en el cual la intensidad del campo eléctrico generado en la zona de depleción es suficiente para generar una avalancha de portadores, es decir, una descarga Geiger.

Como veremos, en el laboratorio se han realizado recorridos de $V_{\text {bias }}$ por encima del Breakdown Voltage con los que podremos determinarlo a partir de los ajustes de la ganancia del detector. Se define el parámetro Over Voltage como la diferencia entre el voltaje de operación $V_{b i a s}$ y el $V_{b r}$ :

$$
V_{\text {over }}=V_{\text {bias }}-V_{b r}
$$

El Over Voltage es realmente el voltaje físicamente relevante. $V_{\text {over }}$ da cuenta de cuánto nos levantamos sobre el Breakdown Voltage. En la parte III veremos que el $V_{b r}$ no es constante con el cambio de temperatura, sino que evoluciona con ella de la misma manera que lo hace $V_{\text {bias }}{ }^{4}$, de tal modo que la propia definición del $V_{\text {over }}$ garantiza, como veremos, su independencia de la temperatura y nos proporciona una información física más pura.

\subsubsection{Modo Geiger y ganancia del SiPM}

Cada microcélula del SiPM está sometida a un $V_{b i a s}$ superior a su $V_{b r}$, de manera que cada vez que un fotón interacciona con un electrón y genera un par electrón-hueco, los portadores son rápidamente acelerados por el campo eléctrico y, en su recorrido, impactan e ionizan al resto de átomos de la red cristalina del semiconductor, aumentando el número de portadores libres. Estos portadores secundarios de carga son también acelerados fuertemente por el campo y pueden seguir ionizando átomos de la red y generando un número creciente de portadores de carga en lo que se conoce como régimen de avalancha o modo Geiger de operación de un fotodiodo. Así, lo que era un solo fotoelectrón atendiendo a la absorción de un solo fotón, imperceptible para la electrónica del experimento, se ha transformado ahora en una avalancha de carga que puede ser detectada, ya que se ha amplificado la señal. No es de extrañar, por tanto, que el $V_{\text {bias }}$ y, por ende, el campo eléctrico creado internamente, influyan fuertemente en la ganancia del $\mathrm{SiPM}$, la cual se define como el ratio entre la carga recogida a la salida, $Q$, y la carga del electrón, $e^{5}$

$$
G=\frac{Q}{e}
$$

\footnotetext{
${ }^{4}$ Algo comprensible realmente, pues $V_{b r}$ ya hemos dicho que no es más que un $V_{b i a s}$ con un cambio de comportamiento del detector.

${ }^{5}$ Carga del electrón $e=1,602 \cdot 10^{-19} C$.
} 
y, además, aumenta con $V_{\text {bias }}$ en una relación lineal

$$
G=p_{1} V_{\text {bias }}+p_{0}
$$

donde $p_{0}$ y $p_{1}$ son parámetros que dependen del dispositivo y de las condiciones de operación (temperatura, etc.).

Cada píxel genera una cantidad de carga altamente uniforme y cuantizada cada vez que se produce una cascada Geiger. Así, la ganancia se alza como un parámetro clave a la hora de definir y caracterizar un fotodiodo de avalancha, de modo que ha sido medida exhaustivamente en nuestro SiPM en el laboratorio, resultados que veremos más adelante en este trabajo en la parte III.

\subsubsection{Quenching}

Para detener la cascada Geiger y detectar el siguiente fotón, se debe reducir notablemente el voltaje de operación mediante un circuito externo al fotodiodo. La técnica utilizada en los SiPM es adosar a las células resistencias llamadas de quenching conectadas en serie a cada fotodiodo. Esto detiene rápidamente la multiplicación en avalancha de portadores mediante la conducción de la carga por la resistencia, la reducción del voltaje por debajo de $V_{b r}$ para detener la multiplicación y la restauración posterior de $V_{\text {bias }}$.

Una característica esencial a la hora de observar la señal en el osciloscopio es que el pulso que observamos debido a la avalancha Geiger es una onda con un risetime corto, mientras que la corriente de salida una vez que la resistencia de quenching ha comenzado a parar la avalancha presenta una forma de pulso con un tiempo de caída relativamente lento, como puede verse en la figura 5 .

\subsubsection{Tiempo de recuperación}

Una vez que un píxel del SiPM ha detectado y medido una señal y se ha generado la avalancha de portadores de carga, la señal debe ser cortada a traves de la resistencia adosada de manera que esa celda vuelva a estar activa para medir un nuevo fotón. El tiempo que requiere realizar el quenching es el llamado tiempo de recuperación y depende mayoritariamente de la capacitancia de la celda y del resistor individual adosado a ella $(\tau \sim R C)$. Los valores típicos de $\tau$ suelen ser de unos $20 \mathrm{~ns}$ para recobrar los píxeles al $100 \%$ para áreas activas de $1 \mathrm{~mm}^{2} \mathrm{y}$

un tamaño del píxel de $25 \mu^{2}$, $50 \mathrm{~ns}$ para un área activa de $1 \mathrm{~mm}^{2}$ y un tamaño del píxel de $50 \mu \mathrm{m}^{2}$ y de 100 a $200 \mathrm{~ns}$ para un área activa de $1 \mathrm{~mm}^{2}$ y un píxel de $100 \mu \mathrm{m}^{2}$. 

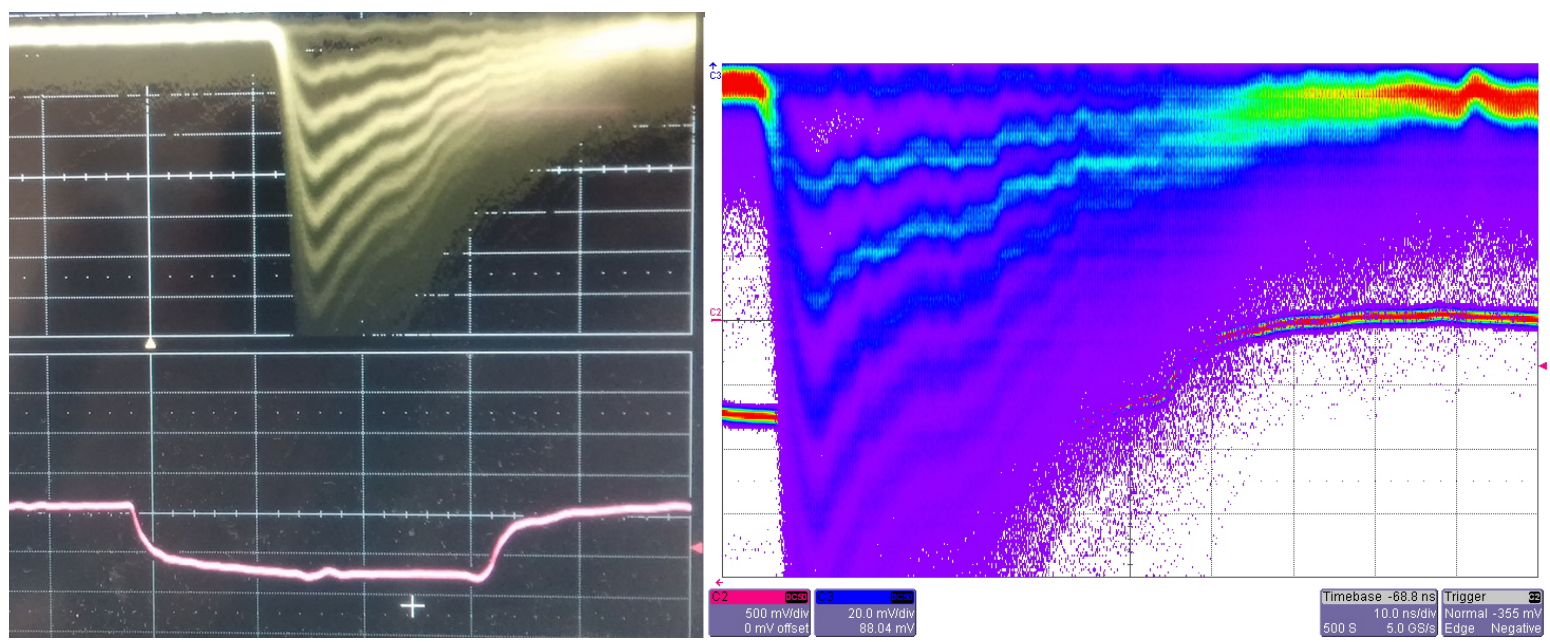

Figura 5: Captura del osciloscopio del laboratorio en modo Analog Persist. La escala vertical mide el voltaje en una escala de $500 \mathrm{mV} /$ división para el trigger y $20 \mathrm{mV} /$ division para la señal del SiPM. La escala temporal horizontal es común a ambas señales, 10 ns/división. El código de colores hace referencia a las zonas más o menos pobladas cuando dejamos que permanezcan dibujadas y persistan en la pantalla. En la parte inferior de cada captura vemos el pulso cuadrado que actuará como ventana de trigger enviada al LED para iluminar y a la QDC para registrar los datos señal (ver la parte II). Izquierda: Pulsos producidos por fenómenos de avalancha en el SiPM cuya carga total ha sido registrada dentro del intervalo de trigger. Como se puede comprobar, el risetime de la señal es mucho más corto que tiempo de bajada controlado por el quenching. Derecha: Detalle de los pulsos recogidos en el que, por encima del ruido, vemos claramente con el Analog Persist la presencia de fingers poblados correspondientes a sucesos de uno, dos, etc. fotoelectrones.

\subsection{Fuentes de ruido en el SiPM}

\subsubsection{Cuentas oscuras (Dark Counts)}

Las cascadas Geiger en cada celda pueden producirse por un fotón incidente y también por la generación espontánea de portadores de carga en la zona de depleción del semiconductor (con un espesor típico de unas pocas micras). Cuando la avalancha la produce un suceso espontáneo, no relacionado con los fotones incidentes que se desea medir, se produce una dark count con un ratio típico de entre $100 \mathrm{kHz}$ y varios $M H z$ por $\mathrm{mm}^{2}$ a $25^{\circ} \mathrm{C}$ y con un umbral de la mitad de la amplitud para un solo fotón, aunque estos valores pueden variar mucho incluso para un mismo tipo de SiPM debido a diferencias en el proceso de fabricación.

Dos procesos son responsables de la ocurrencia de cuentas oscuras:

- Pares $e-h$ generados térmicamente: La energía térmica interna del detector es suficiente como para excitar electrones en los átomos de la red, los cuales pueden ser arrancados y acelerados rápidamente, generando una avalancha debido al $V_{b i a s}>V_{b r}$ aplicado. Lógica- 
mente, esta fuente de ruido se reduce enfriando el SiPM con un ratio de hasta un factor 2 de reducción por cada $8^{\circ} \mathrm{C}$ de descenso de temperatura.

- Generación asistida por el campo: El efecto túnel asistido por posibles trampas en la red cristalina permite la generación sin que medien fonones. Es un efecto minoritario que se reduce al reducir el campo eléctrico en el fotodiodo, lo cual va asociado a una reducción en la ganancia y en la PDE.

Por lo tanto, la caracterización de este ruido natural del detectores una tarea necesaria que ha de realizarse de forma sistemática en cualquier experimento que haga uso de estos dispositivos. Dicha labor de caracterización se llevó a cabo en el laboratorio como parte de este Trabajo de Fin de Máster. Los resultados se exponen en la parte IV.

\subsubsection{Crosstalk}

Mientras sucede una avalancha, los portadores cercanos a las uniones con otras celdas pueden radiar fotones al tiempo que son acelerados por el campo eléctrico. Estos fotones tienden a estar en el infrarrojo, siendo el intervalo de longitudes de onda crítico el que abarca desde $850 \mathrm{~nm}$ hasta $1100 \mathrm{~nm}$. Adicionalmente, pueden viajar distancias considerables dentro del dispositivo. Típicamente, se emiten $2 \cdot 10^{5}$ fotones por cada electrón que cruza la unión. Estos fotones pueden viajar a una celda vecina y, eventualmente, accionar una nueva avalancha Geiger en ella. La probabilidad del Crosstalk se define por tanto como la probabilidad de que la avalancha en una celda produzca una avalancha en una segunda celda ${ }^{6}$. Debido a que el proceso sucede instantáneamente, lo que realmente fue un único fotón puede ser leído por la electrónica como dos, tres o incluso un número más alto de fotones que han llegado simultáneamente a varias celdas del SiPM, distorsionando la medida con señales espúreas. Así, no extraña que la probabilidad de crosstalk deba ser lo más pequeña posible de cara a que el SiPM sea un dispositivo fiable, pues limita considerablemente su resolución en el contaje de fotones.

Es por ello que en este trabajo se realizará dicho estudio detallado en el laboratorio de la probabilidad de crosstalk de nuestro SiPM.

\subsubsection{After-pulse}

El fenómeno conocido como After-pulse (o "pulsos tardíos") se produce cuando algunos electrones generados durante la avalancha son retenidos en trampas y liberados de nuevo tras un cierto intervalo temporal que abarca desde los nanosegundos hasta los microsegundos.

\footnotetext{
${ }^{6}$ La definición será extendida y justificada convenientemente en la parte IV.
} 
Durante una avalancha, un gran número de portadores cruza la unión P-N de tal modo que existe una probabilidad no nula de que algún portador quede atrapado en un estado del gap y sea liberado en los intervalos mencionados accionando un nuevo proceso de avalancha. Cabe mencionar que, si el tiempo que ha estado atrapado el portador ha sido inferior al tiempo de recuperación de la celda, los After-pulse llevan asociada una ganancia inferior. Los estudios preliminares que pueden encontrarse en [5] y otras referencias muestran que los After-pulses no presentan un efecto importante en los SiPM y, por tanto, no merman su rendimiento.

\subsection{Aplicaciones presentes y futuras de los fotomultiplicadores de silicio}

Veamos algunos ejemplos de aplicación actual de los fotomultiplicadores de silicio, así como los objetivos futuros para mejorar los mismos.

En la última década, son cada vez más los experimentos que incorporan fotodiodos de avalancha entre su instrumentación. El calorímetro electromagnético del detector CMS en el LHC del CERN los incorpora en grandes cantidades [6] y son la base de futuros experimentos del CERN como el Colisionador Lineal Internacional (ILC).

Por su parte, el experimento PANDA en FAIR (Darmstadt), en búsqueda de estados exóticos ligados por la interacción nuclear fuerte (como las glueballs), planea la utilización de APD's para la lectura de los cristales de $\mathrm{PbWO}_{4}$ del calorímetro [7]. En experimentos más pequeños, los fotodiodos de avalancha llevan siendo utilizados exitosamente durante años, obteniéndose una gran resolución en energía en la detección de Rayos $\mathrm{X}$ con energías de cientos de $\mathrm{keV}$ [8].

En el ámbito de la medicina, es clave el papel de las Tomografías por emisión de positrones (PET) en el análisis de tejidos y detección de tumores cancerígenos, cuya detección y lectura comienza a realizarse de forma más eficiente en los últimos años gracias a la incorporación de SiPM's en los tomógrafos [9]. Además, se han construído escáneres cerebrales para animales pequeños que están operando con muy buenos resultados [10].

Recientemente, una combinación de PET y Resonancias Magnéticas Nucleares (MRI) basados en detección con SiPM's están proporcionando información sobre metabolismo y funcionalidad con, de nuevo, grandes resultados [11]. Adicionalmente, los errores de paralaje de las PET producidos por la normalmente desconocida profundidad de interacción, están siendo reducidos al mínimo gracias a los llamados AxPET, utilizando SiPM's [12].

Volviendo a los experimentos de física de partículas, el detector de Tokai-to-Kamioka [13], el cual publicó la medida de la desaparición de neutrinos muónicos (que está de acuerdo con experimentos anteriores como MINOS y Super-Kamiokande), siendo, además, la medida más precisa hasta la fecha del ángulo de mezcla $\theta_{23}$, utiliza cerca de 60000 fotodiodos de avalancha. 
Recientemente, se ha demostrado que los SiPM y, en general, los APD's, tienen un futuro brillante como detectores de luz Cherenkov en grandes espectrómetros magnéticos. De hecho, la luz Cherenkov producida en las cascadas de rayos cósmicos altamente energéticos está ya siendo detectada por SiPM's de alta sensibilidad instalados en los telescopios de observatorios como, por ejemplo, Pierre Auger [14].

En otras ramas de la ciencia como en la Física del estado sólido, las técnicas de tipo $\mu S R$ de utilización del espín del muon para estudiar la estructura y procesos dinámicos en el interior de un material a escala atómica, lo cual requiere altos campos magnéticos, han visto altamente simplificada su preparación y montaje experimental gracias a la sustitución de PMT's por SiPM, menos aparatosos, insensibles a los altos campos magnéticos y mucho más eficientes [15].

En el futuro, el objetivo es investigar otro tipo de materiales (aparte del mayoritariamente utilizado silicio) que, manteniendo un coste bajo, repliquen o mejoren las características y eficiencia del silicio en la construcción de LED's, láseres de diodo, detectores, etc. De hecho, es posible la producción de Gain-APD's basados en otros semiconductores con menor ruido, gracias a un mayor gap entre bandas y mayor pureza, alta sensibilidad en el $U V$ y en el azul y menos defectos en la red cristalina que los SiPM, pero no al coste reducido de estos últimos todavía.

En resumen, en el futuro sería ideal la construcción de fotomultiplicadores de avalancha, trabajando en modo Geiger como un SiPM, pero basados en semiconductores con un mayor gap entre bandas que el silicio, alcanzando una mayor pureza intrínseca, menores defectos en la red cristalina del material, reducida producción de fotones secundarios (menor Crosstalk), mejor movilidad de portadores y suficiente vida media de los mismos para poder alcanzar el modo Geiger, etc. 



\section{Parte II}

\section{Experimento. Descripción y métodos.}

Los diversos experimentos se han llevado a cabo en el laboratorio de la Universidad de Granada en el Término Municipal de Ogijares (figura 6), lugar de desplazamiento para cada toma y recogida de datos.

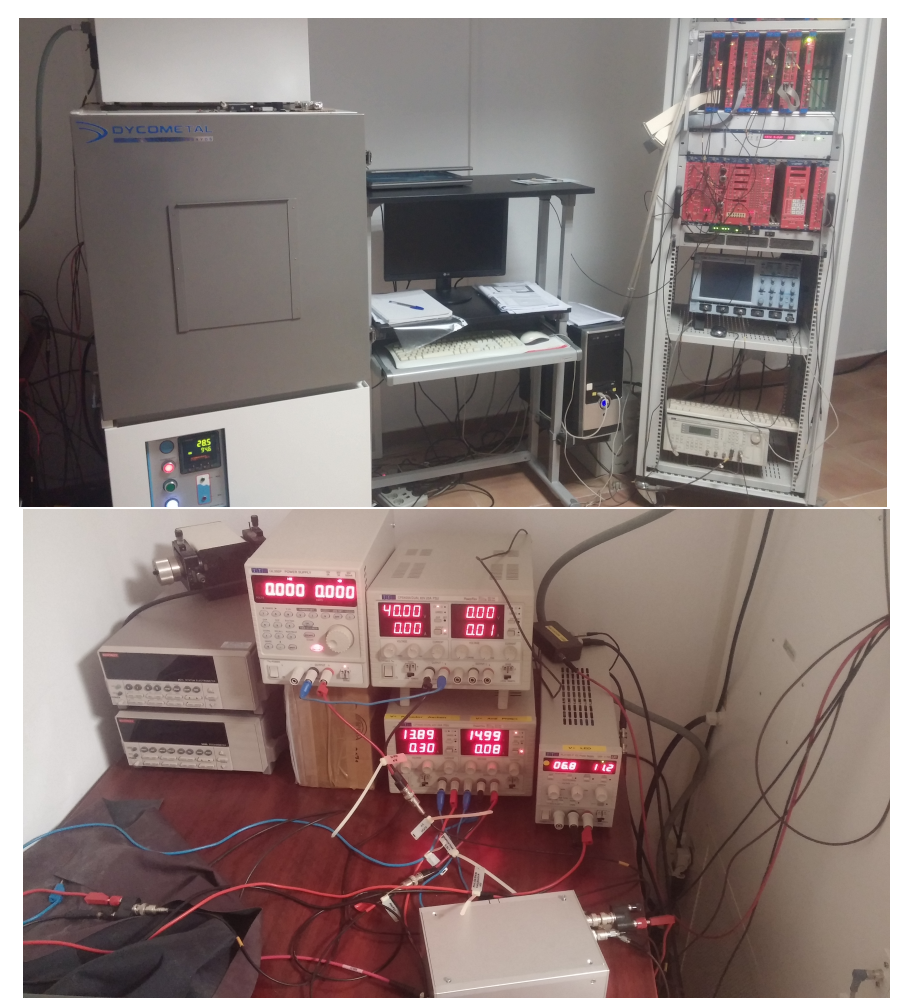

Figura 6: Espacio de trabajo en el laboratorio. Imagen superior y de izquierda a derecha: cámara climática que contiene el SiPM mantenido a unas condiciones controlables de temperatura y humedad, ordenadores de sobremesa y portátil con el software y los códigos necesarios para el control de la cámara, fuentes de alimentación y módulos electrónicos, Rack con los módulos (ver Apéndice A), osciloscopio y generador de funciones. Imagen inferior: Fuentes de voltaje que alimentan al SiPM, al LED de iluminación y al Amplificador Operacional (ver texto).

Por ser los datos más extensos en el tiempo y delicados de tomar, se decidió comenzar por la toma de datos para la determinación de la ganancia del SiPM el día 24 de febrero del 2015. A continuación, se detalla el procedimiento de laboratorio para la recogida de datos a modo de espectros de carga del SiPM para diversas temperaturas de la cámara climática.

Llegados al laboratorio, lo primero que ha de llevarse a cabo es el procedimiento de programación de la cámara climática a la temperatura de trabajo deseada para el run de datos que 
se va a tomar, pues llegar a la temperatura deseada y, sobretodo, estabilizarse en torno a ella con variaciones mínimas, es un proceso que requiere en torno a hora y media en promedio.

Mientras la cámara climática comienza a llevar la temperatura al valor deseado, reduciendo además la humedad interna ${ }^{7}$, debemos preparar el resto de componentes del experimento en función de qué magnitudes se deseen medir. En las siguientes secciones se describe la metodología que se ha seguido en función del objetivo de medida.

\section{Metodología para la determinación de la ganancia}

En esta sección, el objetivo principal será iluminar el SiPM al nivel de un único fotoelectrón. En otras palabras, vamos a preparar el experimento de tal modo que seamos capaces de detectar la carga que se genera en la avalancha del SiPM por la detección de un solo fotón. Para ello, utilizamos un LED cuya emisión en el pico corresponde a una longitud de onda en el azul de $\lambda_{\text {máx }}=480 \pm 5 \mathrm{~nm}$. La luz se lleva hasta la superficie del SiPM mediante fibra óptica. La carga generada será integrada por el módulo electrónico $\mathrm{QDC}^{8}$ en una cierta ventana temporal, que será sincronizada con la señal del SiPM mediante un trigger. Cabe destacar que la ganancia típica de un SiPM $\left(10^{5}-10^{6}\right)$ no es suficiente como para poder ver la señal de un único fotón, sino que debe introducirse una amplificación extra mediante la utilización de un Amplificador Operacional (figura 8) que, en nuestro caso en el laboratorio, amplifica un factor 100.

El SiPM, el Amplificador Operacional y el LED (figura 7) deben ser alimentados con las fuentes (imagen inferior en la figura 6). El SiPM es alimentado con un cierto $V_{\text {bias }}$ que le es suministrado por dos fuentes de tensión en paralelo, una proporcionando 40,0 $\mathrm{V}$ de forma constante y la otra variable y controlada desde el código en el ordenador de sobremesa oscilando entre 28,0 y 30,9 V , según se requiera ${ }^{9}$. El LED debe ser también alimentado para generar un campo eléctrico dentro del diodo que permita la recombinación de portadores que salten el gap de energía, emitiendo los fotones que deben llegar y ser registrados por el SiPM. Para ello, lo someteremos a $6,9 \mathrm{~V}$ y $7,0 \mathrm{~V}$ en varias fases del experimento. Por último, pero no menos importante, se alimenta el operacional con sus habituales $+15,0 \mathrm{~V}$, de tal modo que la débil señal del SiPM en la detección de un solo fotón sea amplificada el factor 100 que se mencionaba previamente.

\footnotetext{
${ }^{7}$ No es un aspecto crucial del experimento, pero se prefiere trabajar con una humedad de en torno al $60 \%$ mejor que con humedades altas de en torno al $95 \%$, que es un valor que suele alcanzarse de forma natural en el interior de la misma cuando se la deja evolucionar libremente fuera de los intervalos de toma de datos y puede producir condensación.

${ }^{8}$ Ver Apéndice A. La QDC recoge una carga señal y la integra en una cierta ventana que le indica el Dual Timer, transformándola en una señal digital que podemos leer e interpretar con el ordenador.

${ }^{9}$ Voltaje de bias total que alimenta el SiPM entre 68,0 y 70,9 $\mathrm{V}$.
} 


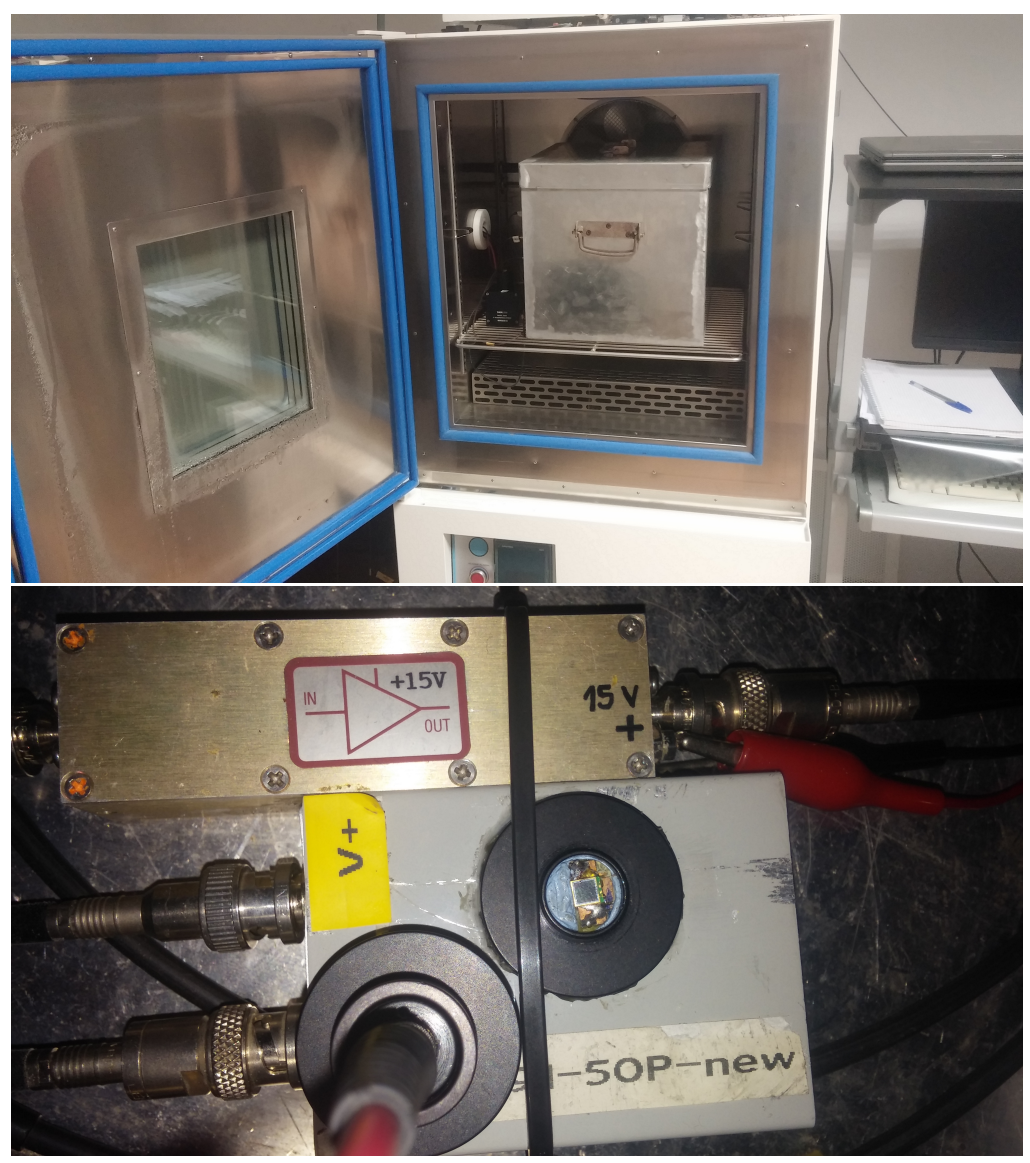

Figura 7: Imagen superior: Detalle del interior de la cámara climática. El detector, el LED y el Amplificador Operacional se encuentran dentro de la caja metálica como medida de asilamiento adicional para reducir el background o ruido ambiente. Imagen inferior: Interior de la caja metálica. En la parte superior se encuentra el Amplificador Operacional y, cerca de la parte central de la imagen, se encuentra el SiPM (instrumento de forma cuadrada y con dimensiones reducidas, $3 \times 3 \mathrm{~mm}^{2}$ ).

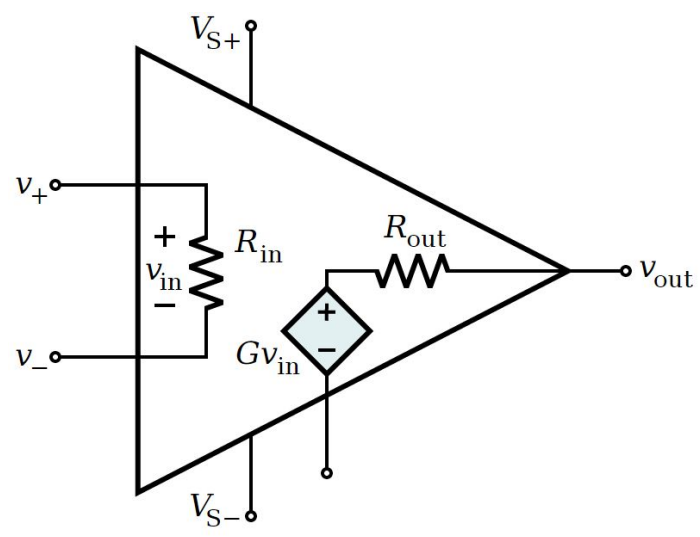

Figura 8: Circuito equivalente de un amplificador operacional. 
En el estado actual no estamos en condiciones de realizar ningún experimento con sentido todavía. Debemos decirle al LED cuándo debe iluminar al SiPM, y a la QDC cuándo debe leer los datos del continuo de señales y ruido que se generan en el tiempo. Queremos que, en la misma ventana temporal, el LED ilumine el SiPM y el módulo de la QDC lea lo que éste le está diciendo. Para ello, vamos a utilizar un generador de funciones como el de la figura 9. Lo que vamos a hacer es generar una señal tipo pulso cuadrado en un canal del generador que vamos a clonar en un segundo canal de salida. Uno de los canales nos permitirá enviar la señal al LED, el cual iluminará durante intervalos temporales de anchura y periodicidad las del pulso. La misma señal, pero clonada y llevada por el segundo canal del generador, será enviada al módulo del Dual Timer.

En el Apéndice A se encuentran descripciones breves y especificaciones de los módulos electrónicos, basta saber aquí que el Dual Timer recibe una señal NIM y produce una nueva señal NIM a la cual se le pueden ajustar tanto el desfase como la anchura temporal. Con ello, creamos una ventana de trabajo que, llevada ahora al módulo de la QDC, permitirá a ésta última saber cuándo y durante cuánto tiempo debe leer los datos de carga del SiPM, integrar dicha carga y producir la señal digital proporcional que podrá interpretarse en el ordenador.

En definitiva, el pulso cuadrado de periodo $250 \mu s$ (frecuencia $4 \mathrm{kHz}$ ) y anchura temporal 25 ns actuará de trigger para ambos instrumentos, disparando un flash de luz en el LED durante esa ventana temporal e indicando a la QDC cuándo y por cuánto tiempo debe comenzar y continuar registrando datos. En concreto, el pulso cuadrado procedente del generador y que queremos llevar a la QDC se hace pasar por el módulo Dual Timer (ver Apéndice A), con el cual mantenemos su frecuencia pero modificamos su anchura temporal de $25 \mathrm{~ns}$ a $80 \mathrm{~ns}$, de tal modo que la QDC puede abarcar con creces toda la señal del SiPM. Puede visualizarse este pulso cuadrado en la parte inferior de la figura 5 izquierda claramente y solapado con las señales en la captura de la derecha en esa misma figura.

Si se han realizado los pasos anteriores correctamente, el SiPM debería estar ya alimentado con un cierto $V_{\text {bias }}$ y recibiendo, con la periodicidad del trigger creado por el generador de funciones, fotones procedentes del LED que esperamos que produzcan en alguna de las celdas la creación de pares portadores que serán acelerados por el campo eléctrico interno y se multiplicarán en avalancha, generando una señal que la QDC registrará. 


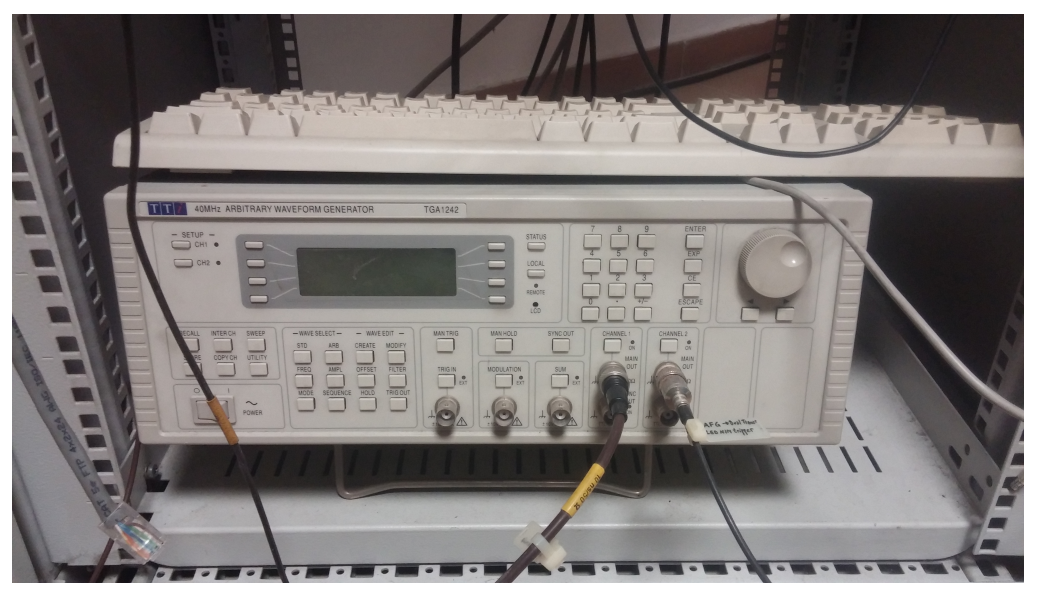

Figura 9: Detalle del generador de funciones visible en la parte inferior del Rack de la imagen de la izquierda en la figura 6.

Sin embargo, antes de lanzar el run que nos interese debemos asegurarnos de que esto sea así y debemos, además, buscar un voltaje de bias $\left(V_{\text {bias }}\right)$ que sea superior al Breakdown Voltage $\left(V_{b r}\right)$ para que el SiPM trabaje en el modo Geiger que buscamos.

Para llevar a cabo estas verificaciones, lo más conveniente es realizar un visionado a tiempo real en el osciloscopio (figura 5) sin más que llevarnos la señal del SiPM y del trigger a los canales del mismo.

Que veamos o no la señal depende en gran medida del buen hacer en el laboratorio y del cuidado con el que el experimento ha sido preparado. Una preparación descuidada puede desbaratar días de toma de datos en los que la señal no se comporta como sería físicamente esperable por ruidos y acoplamientos estocásticos entre señales.

Si vemos la señal en el osciloscopio, sea a la primera o tras corregir todos los problemas, es momento de determinar el intervalo de $V_{\text {bias }}$ en el que vamos a tomar datos. El criterio utilizado es comenzar la toma de datos en voltajes inferiores a la vista del primer pico de un fotoelectrón, de tal modo que los primeros runs que tomaremos nos mostrarán sólo un 'pedestal' correspondiente a ruido electrónico aleatorio. El límite superior será determinado por el voltaje para el cual el visionado de los espectros de carga contenga tantos sucesos que se torne imposible realizar un análisis preciso. Ejemplos de ambos casos extremos se muestran en la figura 10.

La determinación de los extremos de $V_{\text {bias }}$ en cada toma de datos para cada temperatura es un paso crucial no por la importancia de los mismos en el análisis que vamos a realizar, sino porque los datos interesantes para los cuales el SiPM trabaja cómodamente para $V_{b i a s}>V_{b r}$, es decir, en modo Geiger, se encuentran entre ambos extremos y es donde queremos realizar el muestreo. 
Con este fin, nos llevamos los cables que transportan la señal del SiPM y el trigger desde el osciloscopio de vuelta a la QDC y lanzaremos el run con el código del ordenador de sobremesa, que se comunicará con los módulos y las fuentes de alimentación y creará un fichero de datos con la carga recogida en $f C$ en abcisas y las cuentas obtenidas en cada bin en ordenadas, tal y como se muestra en la figura 10.

Lo dejamos tomar datos durante un minuto ${ }^{10}$ y visionamos los espectros de carga que obtenemos. El proceso de búsqueda de los extremos ha de ser realizado con precaución y paciencia, pero es relativamente rápido.
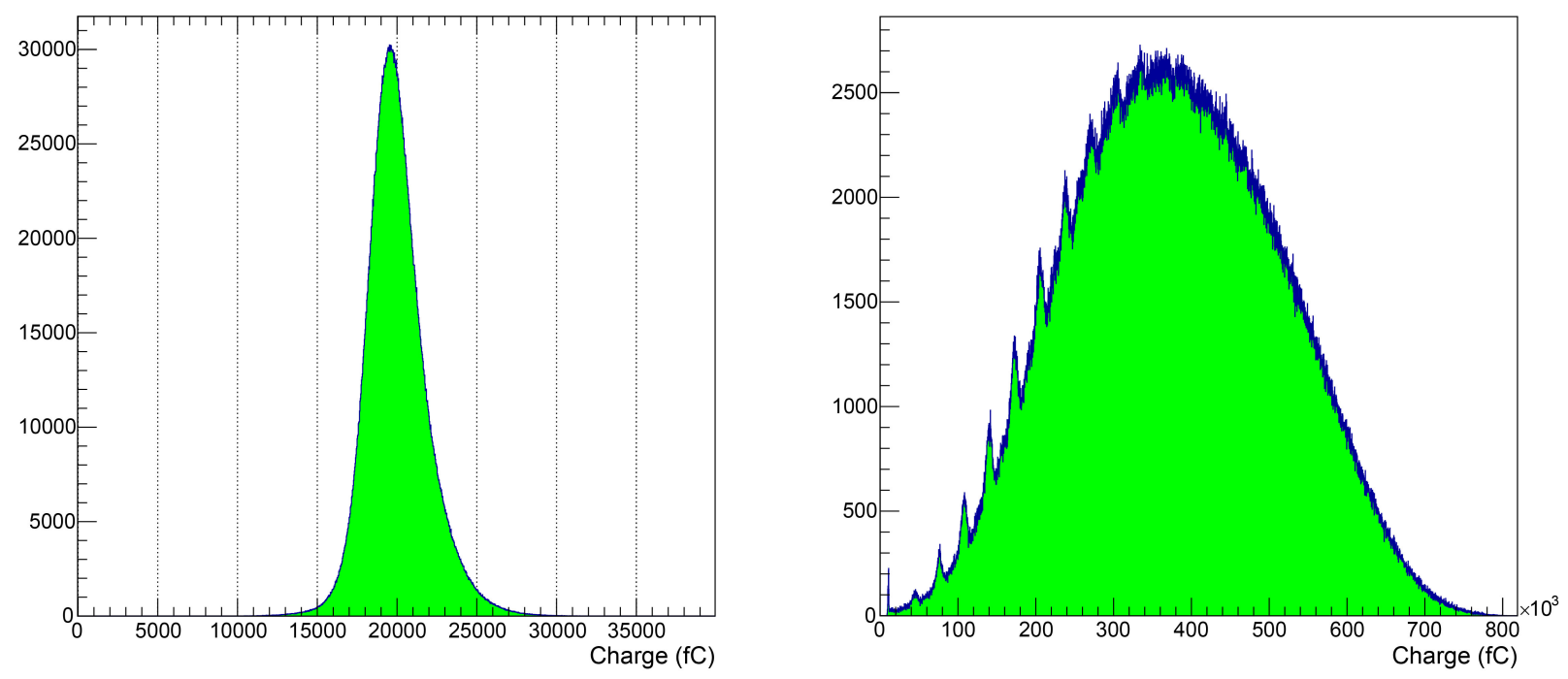

Figura 10: Espectros de carga recogidos con la QDC para una temperatura $T=0{ }^{\circ} \mathrm{C}$ y un voltaje del LED $V_{L E D}=6,9 \mathrm{~V}$. La carga en $f C$ se muestra en abcisas, y el número de cuentas en ordenadas. Izquierda: límite inferior en el voltaje de bias para la toma de datos, $V_{\text {bias }}=68,5 \mathrm{~V}$. Sólo el ruido electrónico y estocástico es visible. Derecha: límite superior en el voltaje de bias para la toma de datos, $V_{\text {bias }}=70,3 \mathrm{~V}$. Nótese el cambio en la escala horizontal desplazada hasta un orden de magnitud indicando la enorme cantidad de portadores que ahora forman la avalancha. Los picos de fotoelectrones, en la mitad izquierda del pulso, se tornan casi indistinguibles al solaparse la gran cantidad de sucesos simultáneos, indicando que estamos en el límite de $V_{\text {bias }}$.

Determinados los extremos y funcionando todos los componentes correctamente, como se debe haber comprobado con estos pasos, es momento de lanzar un run de datos definitivo para la temperatura que hemos seleccionado en la cámara climática especificando en el código el muestreo en $V_{\text {bias }}$ que deseamos realizar, entre los extremos determinados, y el tiempo de

\footnotetext{
${ }^{10}$ Tiempo suficiente pues, a pesar de no recoger tanta estadística como necesitaremos para el run final, hay sucesos suficientes como para determinar bien los límites. Recuérdese que la frecuencia del trigger es de $4 \mathrm{kHz}$ de modo que, en $60 \mathrm{~s}$, tenemos en torno a 240000 triggers.
} 
toma de datos para cada $V_{\text {bias }}$. Además, seleccionaremos los voltajes de $V_{L E D}$ para los cuales deseamos realizar un run.

Para cada temperatura y voltaje del LED, se ha muestreado desde el extremo inferior al extremo superior de $V_{\text {bias }}$ en intervalos de $0,1 \mathrm{~V}$, obteniendo para cada punto un espectro de carga diferente. El tiempo de toma de datos en cada valor de $V_{\text {bias }}$ es de $120 s$ y se han realizado los runs para dos valores de $V_{L E D}$, que son 6,9 y 7,0 $\mathrm{V}$, duplicando esto el tiempo de toma de datos. Además, realizamos este procedimiento en cada ocasión para tomar datos a valores de temperatura $T=-10,-5,0,5,10^{\circ} \mathrm{C}$ que se verán y analizarán en la parte III.

\section{Metodología para la determinación de las cuentas oscu- ras y el Crosstalk}

La filosofía del experimento cambia completamente en esta segunda parte. Nuestro objetivo se desplaza de medir la respuesta del SiPM a estimulos externos, para determinar su bondad como detector de partículas elementales como los fotones, a medir el ruido intrínseco característico de este tipo de detector de estado sólido. Previamente (sección 1.6) se ha explicado cómo la generación térmica aleatoria de un par $e-h$ acelerado por el campo eléctrico interno puede llevar a una celda del SiPM a una avalancha espúrea indistinguible de un suceso de señal real. Además, los portadores de la avalancha pueden radiar, afectando a otras celdas vecinas y activando nuevas avalanchas no deseadas mediante el fenómeno conocido como Crosstalk $^{11}$.

Medir las cuentas oscuras en el SiPM pasa por desconectar cualquier tipo de iluminación externa, de modo que desconectamos para esta fase del experimento el generador de funciones y el LED. Queremos un SiPM aislado que, de enviar alguna señal, estemos completamente seguros que se debe a un suceso de su ruido intrínseco. Al seguir estando conectado el SiPM a las dos fuentes en paralelo que lo alimentan con un cierto $V_{b i a s}$, la señal que él envía es un contínuo de ruido electrónico y sucesos de avalancha por portadores creados aleatoriamente por agitación térmica y acelerados por el campo eléctrico interno generado por dicho voltaje de bias. El procedimiento seguido en esta parte se describe a continuación.

La señal del SiPM no es llevada a la QDC ahora, no estamos interesados en recoger la carga total recibida y llevarla a una señal digital. Lo que nos interesa ahora es conocer la cantidad de sucesos que ocurren para determinados niveles de voltaje, esto es, queremos saber cuantos sucesos espúreos se registran como uno, dos, tres, etc. fotoelectrones simultáneos en diversas celdas. Para ello, la señal del SiPM ha de ser pasada por un veto, un discriminador que varíe el

\footnotetext{
${ }^{11}$ Algo que sucede independientemente de si el suceso procede de la señal física o de un evento estocástico de ruido, pero es aquí donde vamos a tomar los datos necesarios que analizaremos en la parte IV.
} 
voltaje umbral por debajo del cual rechazamos los sucesos. Así, la señal es llevada al módulo LTD $^{12}$ que nos permitirá realizar runs variando el voltaje umbral (si volvemos a la figura 5, el LTD haría evolucionar el voltaje umbral de arriba a abajo, desechando cada vez más sucesos a medida que nos vamos a sucesos que involucran más fotoelectrones).

Realizado el veto, todavía necesitamos contar cuántos sucesos han superado el umbral, tarea que realizará el módulo del Scaler, por lo que a él nos llevaremos la señal tras pasar por el discriminador. Tras un run siguiendo esta metodología, podremos graficar el número de sucesos registrados por el Scaler en función del voltaje umbral dinámico del LTD. Por lo visto en la figura 5, esperamos recoger cada vez menos sucesos a medida que el voltaje umbral crece.

Gracias a la fase anterior del experimento, tenemos para cada una de las temperaturas que se han estudiado los límites de $V_{\text {bias }}$ que delimitan los intervalos para los cuales el SiPM trabaja en modo Geiger. De tal modo que, para cada temperatura, nos situaremos en el extremo inferior de $V_{\text {bias }}$ de nuevo para comenzar los runs y realizaremos una toma de datos con el LTD y el Scaler en intervalos de 0,4 V del voltaje de bias. Se realizaron un total de cinco runs de datos para cada temperatura, abarcando el intervalo de $V_{\text {bias }}$ de en torno a $0,9 V$ en el que el SiPM trabaja en modo Geiger cómodamente que veíamos en la sección anterior.

La razón del incremento en el paso tomado para $V_{\text {bias }}$ desde $0,1 \mathrm{~V}$ en la sección anterior a los $0,4 \mathrm{~V}$ actuales radica en el hecho de que vamos a tomar datos para 250 voltajes umbrales en el LTD, abarcando un intervalo desde $0 \mathrm{mV}$ hasta $-250 \mathrm{mV}$ en los que el Scaler medirá el número de cuentas registradas durante $120 \mathrm{~s}$ por cada voltaje umbral para recopilar una estadística considerable. Este tiempo, junto a los cinco runs que se van a realizar para abarcar todo el intervalo de $V_{\text {bias }}$ dan lugar a una toma de datos global para cada temperatura de en torno a $33 h$, por lo que un paso incluso inferior del voltaje de bias no sólo no va a aportar una mejor información, sino que disparará el tiempo de toma de datos demasiado.

Para esta fase, tomaremos los runs de datos para las temperaturas $T=-10,0,10^{\circ} \mathrm{C}$, pues veremos en la parte IV que aportan toda la información necesaria con creces.

\footnotetext{
${ }^{12}$ Low Threshold Discriminator. Ver Apéndice A.
} 


\section{Parte III}

\section{Resultados para la Ganancia}

En esta parte se van a exponer y discutir los resultados obtenidos para el espectro de carga, la ganancia del SiPM, así como el resto de magnitudes derivadas de su medida, como el Breakdown Voltage, $V_{b r}$, o el Over Voltage, $V_{\text {over }}$.

Una vez recogidos los archivos de datos desde el PC del laboratorio, se procede a su visionado con el paquete de análisis de datos ROOT para comprobar la corrección de los mismos y descartar cualquier tipo de error en la puesta a punto de los instrumentos. Para ello, es muy ilustrador el estudio de los datos en bruto que la QDC arroja: los espectros de carga recogidos.

Cada fotón que logra crear un par $e-h$ en la red cristalina del silicio genera, como se ha explicado previamente, una avalancha en la que se multiplican los electrones creados, amplificando con una cierta ganancia caracterítica del detector la carga recogida. En base a este sencillo principio, una visualización de los espectros de carga como los de la figura 11 nos permite directamente identificar las cuentas obtenidas en un cierto bin (intervalo) de carga, lo cual nos permite reconocer la llegada y detección de un fotón en una celda, produciendo una señal referente a la recolección de la carga de un fotoelectrón amplificada según la ganancia del detector y, de forma equivalente, podremos ver los picos referentes a la llegada de dos fotoelectrones a dos celdas diferentes de forma simultánea, a la llegada simultánea de tres, etc.

Comentemos toda la información que puede extraerse de la figura 11, donde se muestran las comparaciones entre los espectros de carga medidos con la QDC a $T=-5^{\circ} \mathrm{C}$ para valores de $V_{L E D}$ de $6,9 \mathrm{~V}$ y $7,0 \mathrm{~V}$. Adicionalmente, se realiza este análisis de datos para dos $V_{\text {bias }}$ diferentes, $68,7 \mathrm{~V}$ (izquierda) y 70,1 $\mathrm{V}$ (derecha), con el fin de ilustrar los fenómenos físicos relevantes. Pueden obtenerse estas comparaciones para cualquier temperatura y para cualquier $V_{\text {bias }}$. En la figura se muestra un caso concreto y muy ilustrativo que se ha obtenido en el laboratorio como complemento a la explicación.

Previa a la explicación del comportamiento de los espectros es la explicación del primer pico que llamamos pedestal. Recordemos la figura 5: en ella, puede observarse una señal importante procedente del ruido electrónico del detector e instrumentación que está en la base de las medidas, es el background de señales espúreas que en el osciloscopio vemos como una zona muy poblada en torno a $0 \mathrm{~V}$ y que la QDC recoge y nos muestra a modo de pico pedestal sin información física relevante en el cometido que se está abordando en esta parte.

En los espectros podemos ver los picos o fingers relativos a la detección de uno o varios fotones simultáneamente. Recordemos que los fotones que recibe el SiPM proceden del LED que opera con un potencial que podemos controlar, de modo que, si incrementamos el voltaje 
aplicado al mismo, aumenta la cantidad de portadores que saltan entre las bandas de valencia y de conducción, de modo que, de forma efectiva, estamos aumentando la cantidad de fotones emitidos por el LED que iluminan el SiPM y, con ella, la probabilidad de detectar varios fotones simultáneamente. Por lo tanto, no extraña encontrar en las comparaciones que aumentar el voltaje del LED de $6,9 \mathrm{~V}$ a 7,0 $\mathrm{V}$, dejando la temperatura y el $V_{\text {bias }}$ inalterados, produce una mayor población de los eventos de recogida simultánea de varios fotones, siendo menos probable la recogida de cero, uno o pocos fotones simultáneamente.

Adicionalmente, podemos realizar exactamente el mismo análisis pero aumentando el $V_{\text {bias }}$ de $68,7 \mathrm{~V}$ a $70,1 \mathrm{~V}$. Ahora el campo eléctrico inducido es mayor, de modo que las avalanchas involucran a un número de portadores mucho mayor y la QDC recoge mucha más carga. En otras palabras, la ganancia del detector aumenta de la mano del $V_{b i a s}$, cosa que se explicará a continuación en el análisis de las siguientes figuras. Nótese de hecho el cambio en la escala horizontal entre la gráfica de la izquierda y la de la derecha en la figura 11, al permitirnos la QDC graficar los datos a una escala grande o pequeña.
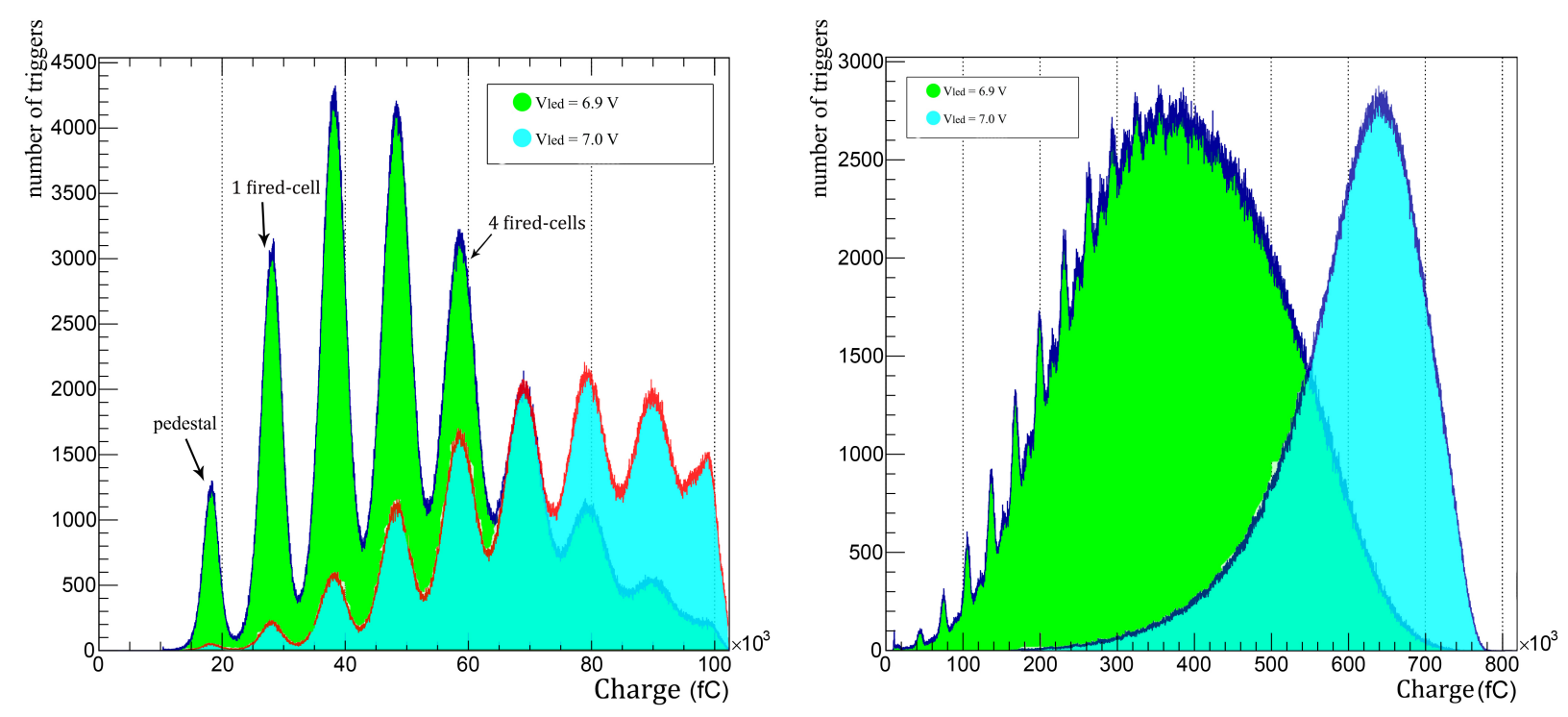

Figura 11: Espectros de carga del SiPM medidos con la QDC a $T=-5^{\circ} \mathrm{C}$ para dos valores de $V_{L E D}$. Izquierda: $V_{\text {bias }}=68,7 \mathrm{~V}$. Derecha: $V_{\text {bias }}=70,1 \mathrm{~V}$. 


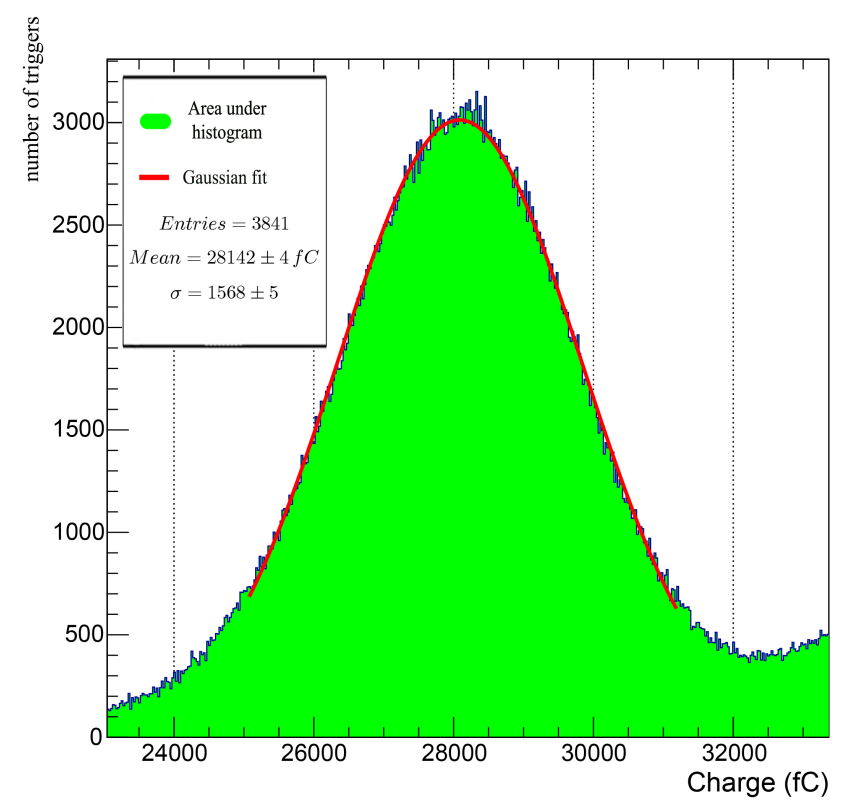

Figura 12: Detalle del ajuste gaussiano realizado a los picos. Ejemplo concreto para el pico de un fotoelectrón en el espectro de carga de la gráfica de la izquierda en la figura 11. La línea sólida muestra el resultado del ajuste gaussiano al histograma de datos.

Pasemos ahora a mostrar y analizar los resultados obtenidos para la evolución de la ganancia del SiPM con el voltaje de bias. Primeramente, debemos calcularnos dicha ganancia, procedimiento que se explica seguidamente. Atendamos a la figura 12.

Nuestro objetivo, una vez reconocido el origen físico de cada pico y su identificación como un (varios) fotón (fotones) creando un fotoelectrón (fotoelectrones simultáneos en diversas celdas), es averiguar la carga que se ha recogido a partir de la generación de un sólo fotoelectrón con el objetivo de conocer la ganancia total del SiPM. Para ello, es acertado el siguiente procedimiento.

Ajustamos a cada finger una gaussiana (puede comprobarse en la figura 12 la buena bondad del ajuste ${ }^{13}$ ), cuya media nos indica, con un muy estrecho margen de incertidumbre, la carga total recogida por el evento.

Si realizamos el ajuste para los dos primeros picos señal en cada histograma, dejando fuera el pedestal de ruido electrónico, podemos calcularnos la diferencia entre la carga recogida para dos fotoelectrones y la recogida para uno sólo, pues son los que hacen referencia a sucesos físicos de detección de fotones. Mediante esta diferencia, eliminamos los desplazamientos electrónicos y desfases de la señal y nos quedamos con un dato que hace referencia a la carga total recogida en

\footnotetext{
${ }^{13}$ Nuevamente, en la figura se muestra un caso concreto y muy ilustrativo como complemento a la explicación para una temperatura de $T=-5^{\circ} \mathrm{C}$ y un $V_{\text {bias }}=68,7 \mathrm{~V}$.
} 
el SiPM a partir de la generación de solo un fotoelectrón primario, de donde es inmediato inferir el valor de la ganancia para esa temperatura y $V_{\text {bias }} \sin$ más que descontar la amplificación por un factor 100 del Amplificador Operacional, presente en el circuito electrónico del experimento, dividiendo por ese factor, y normalizando finalmente a la carga del fotoelectrón primario.

Hecho esto, puede obtenerse para cada temperatura la evolución de la ganancia en función de $V_{\text {bias }}$. En la figura 13 se muestran en detalle los datos experimentales obtenidos para una temperatura de $-10^{\circ} \mathrm{C}$ y el ajuste lineal realizado a los mismos. Como vemos, la ganancia presenta con el voltaje de bias una dependencia claramente lineal que se ha ajustado de acuerdo a la ecuación 5, que recordamos aquí:

$$
G=p_{1} V_{b i a s}+p_{0}
$$

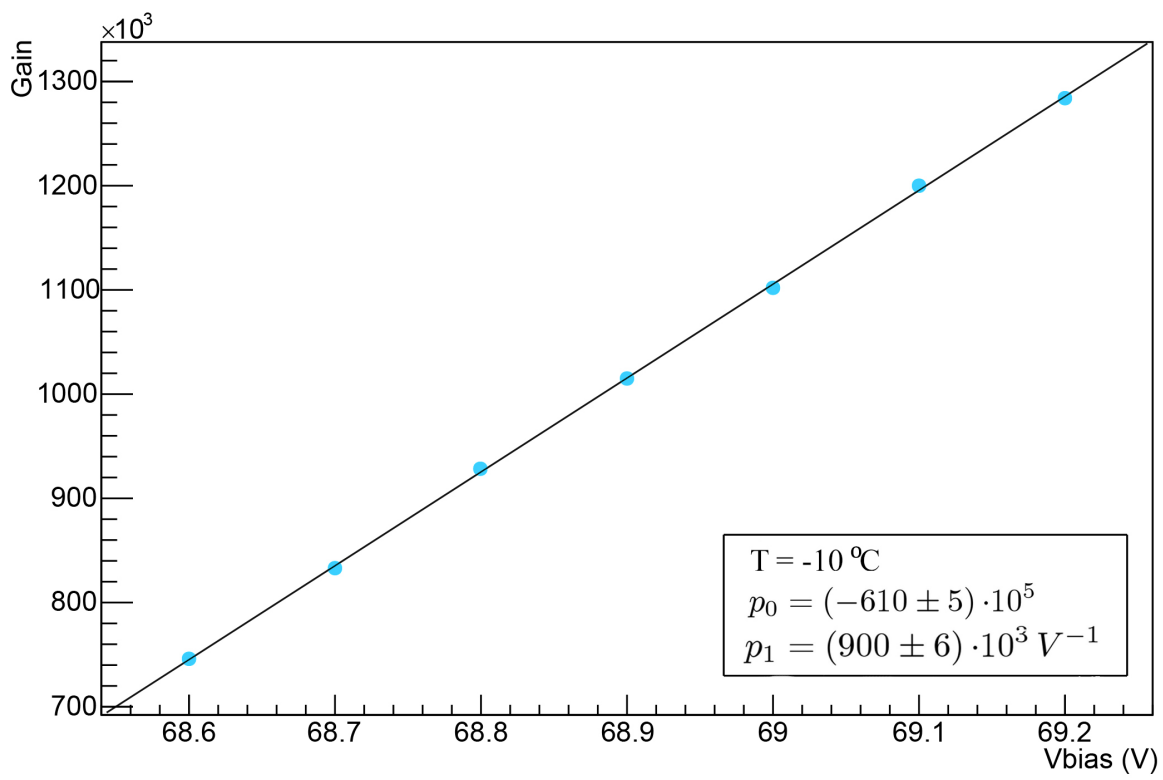

Figura 13: Resultados experimentales y ajuste lineal de la ganancia del SiPM en función del $V_{\text {bias }}$ para $T=-10^{\circ} \mathrm{C}$. Los puntos azules representan los datos obtenidos y la línea sólida negra es el ajuste realizado a dichos datos de acuerdo con la ecuación 5. Los parámetros obtenidos del ajuste se muestran en la esquina inferior derecha. Los intervalos de incertidumbre de las medidas son órdenes de magnitud inferiores a los valores obtenidos, por ello no son representados.

En la figura 14 se representan todos los datos experimentales obtenidos para la ganancia en función de $V_{\text {bias }}$ para cada una de las temperaturas estudiadas en el laboratorio así como los ajustes lineales realizados a los mismos. Los parámetros de los ajustes para cada temperatura se adjuntan en la tabla 2. 
Es interesante y necesario detenerse brevemente en la figura 14, pues observamos una tendencia de la ganancia a desplazarse a $V_{\text {bias }}$ más grandes con la subida de la temperatura. En las siguientes figuras que vamos a analizar comprobaremos que esto es simplemente debido a un incremento del Breakdown Voltage con la temperatura.

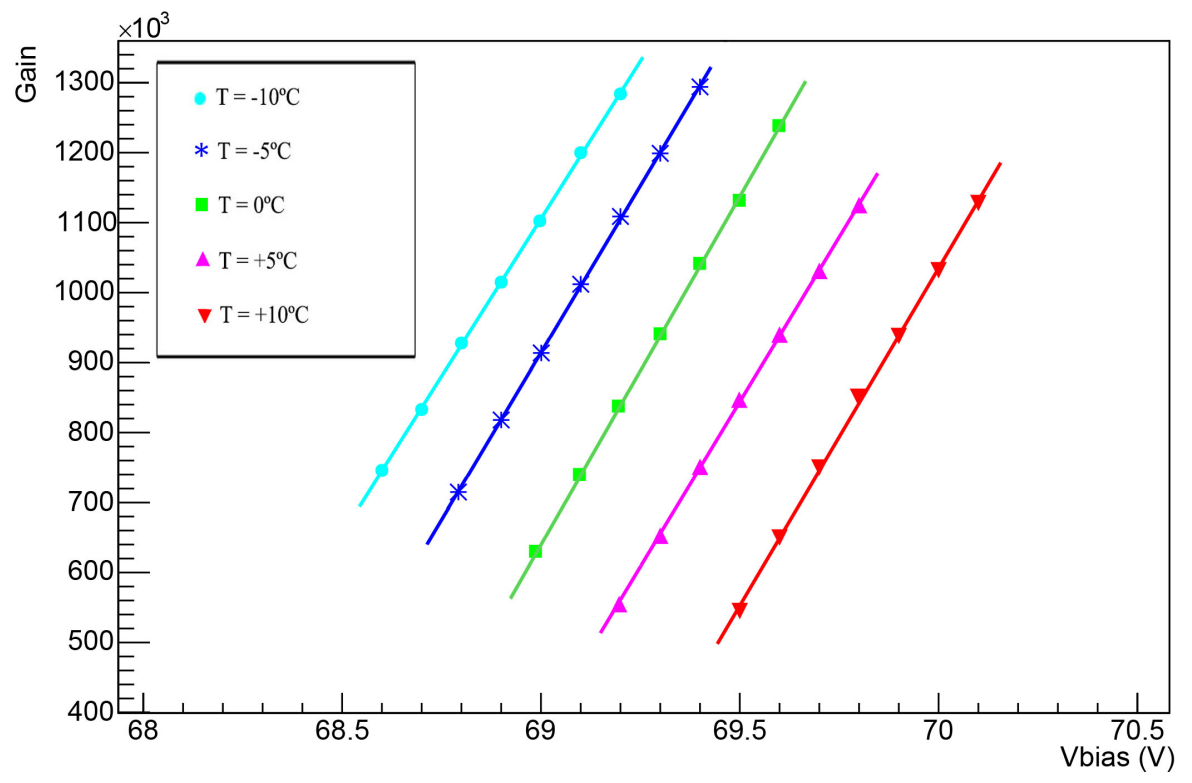

Figura 14: Resultados experimentales de la dependencia de la ganancia con la temperatura para todas las temperaturas a las que se ha trabajado (ver leyenda). Se muestran los diversos ajustes lineales para cada una de las temperaturas, cuyos parámetros quedan recogidos en la tabla 2. Por claridad, se muestran los ajustes del mismo color que los datos experimentales a los que hacen referencia. Los intervalos de incertidumbre de las medidas son órdenes de magnitud inferiores a los valores obtenidos, por lo que no son representados.

\begin{tabular}{|c|c|c|}
\hline Temperatura $\left({ }^{\circ} \mathrm{C}\right)$ & $p_{1} \cdot 10^{3} \mathrm{~V}^{-1}$ & $p_{0} \cdot 10^{5}$ \\
\hline \hline-10 & $900 \pm 6$ & $-610 \pm 5$ \\
\hline-5 & $955 \pm 4$ & $-650 \pm 3$ \\
\hline 0 & $996 \pm 7$ & $-681 \pm 5$ \\
\hline 5 & $942 \pm 6$ & $-646 \pm 4$ \\
\hline 10 & $965 \pm 10$ & $-665 \pm 7$ \\
\hline
\end{tabular}

Cuadro 2: Parámetros obtenidos para los ajustes lineales del tipo $G=p_{1} V_{\text {bias }}+p_{0}$ de la figura 14.

Una vez obtenidos los resultados y ajustes de la ganancia en función del voltaje de bias para cada temperatura, estamos en condiciones de inferir el voltaje a partir del cual el SiPM entra en modo Geiger que, como se ha comentado previamente, se conoce como Breakdown Voltage 
$\left(V_{b r}\right)$. Por definición, se trata del voltaje en el cual el SiPM presenta una ganancia nula (es el valor de cambio a un régimen de operación en modo avalancha), de modo que obtendremos el $V_{b r}$ para cada temperatura extrapolando linealmente a cero la ganancia:

$$
\begin{gathered}
G=0=p_{1} V_{b r}+p_{0}, \\
V_{b r}=-p_{0} / p_{1} .
\end{gathered}
$$

Los resultados obtenidos se muestran en la figura 15.

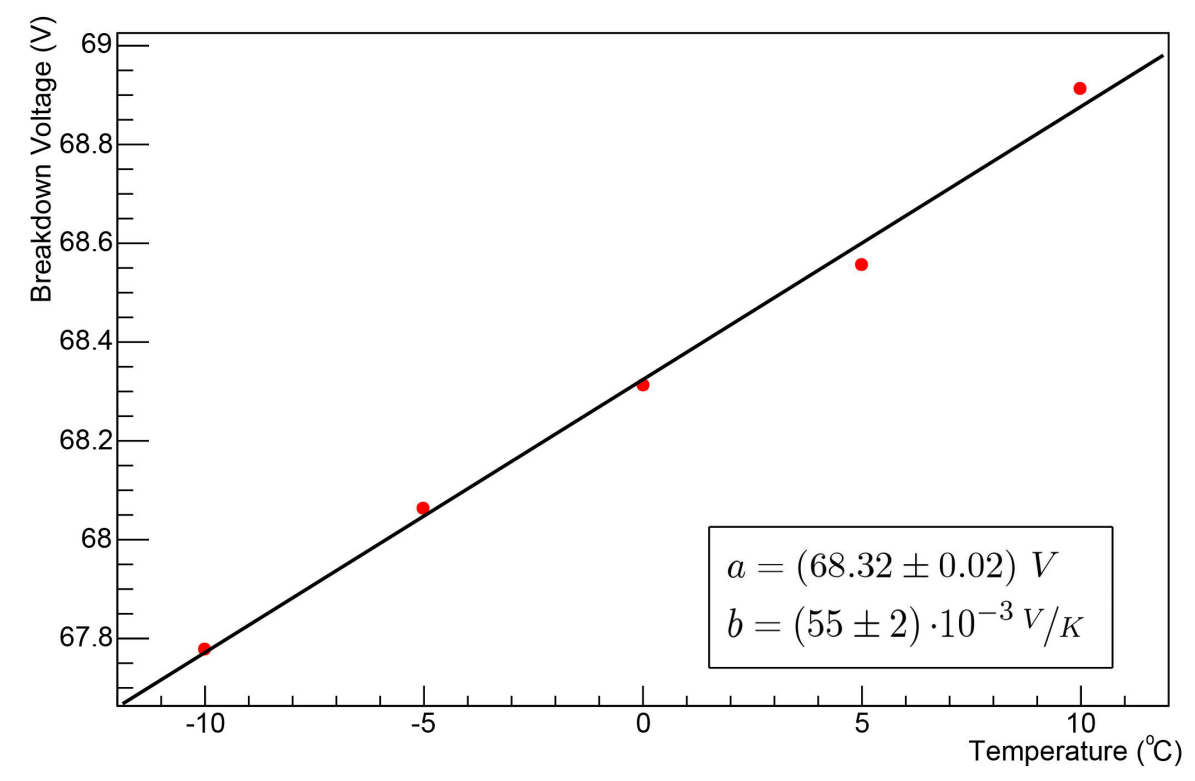

Figura 15: Resultados experimentales para la dependencia del Breakdown Voltage, $V_{b r}$, con la temperatura. Los puntos representan los datos obtenidos en laboratorio y la línea sólida negra representa el ajuste lineal de los mismos. En la esquina inferior derecha se adjuntan los resultados obtenidos para dicho ajuste del tipo $V_{b r}=b \cdot T+a$.

Como puede comprobarse, el $V_{b r}$ crece linealmente con la temperatura, efecto que podíamos intuir y que explica el desplazamiento a altos $V_{\text {bias }}$ de los valores de la ganancia cuando aumentábamos la temperatura en la figura 14. El ajuste realizado e incluido en la leyenda de la gráfica es del tipo

$$
V_{b r}=b \cdot T+a=(55 \pm 2) \cdot 10^{-3} T+(68,32 \pm 0,02)[V]
$$

donde $a$ es la ordenada en el origen que, de forma esperada, recupera el valor de $V_{b r}=68,3 \mathrm{~V}$ para $T=0^{\circ} \mathrm{C}$ dentro de sus márgenes de incertidumbre. 
Podemos, ahora, preguntarnos qué ocurre si eliminamos los efectos de la temperatura mediante la representación de los resultados obtenidos para la ganancia, ya no frente a $V_{\text {bias }}$, sino frente al Over Voltage, que es la magnitud que contiene verdadero sentido físico al indicarnos cuánto nos estamos levantando sobre el Breakdown Voltage, que marca la entrada en modo Geiger del SiPM (ecuación 3) para cada T, librándonos así de la dependencia con la misma en la representación.

Como puede comprobarse en la figura 16, se produce el colapso esperable de todos los resultados que se han obtenido hasta ahora. La ganancia del detector frente a $V_{\text {over }}$ presenta una dependencia lineal independiente de la temperatura.

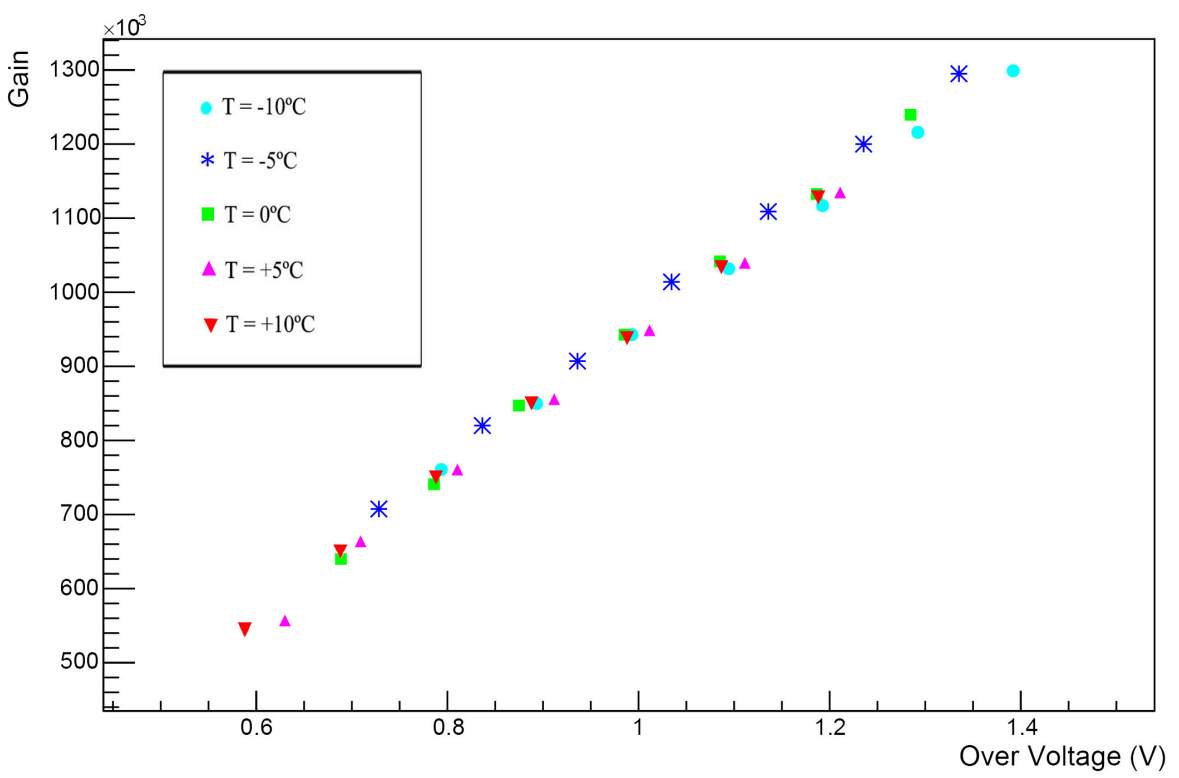

Figura 16: Resultados obtenidos para la dependencia de la ganancia con el Over-Voltage para cada una de las temperaturas de operación con las que se ha trabajado. Los distintos marcadores y el código de colores vienen recogidos en la leyenda de la esquina superior izquierda.

Los estudios anteriores caracterizaron la ganancia del SiPM en función de $V_{\text {bias }}$ y de la temperatura de modo que, en un experimento real y conocidos los voltajes de operación y la temperatura del dispositivo, el factor de amplificación estaría determinado. 



\section{Parte IV}

\section{Resultados para cuentas oscuras y Crosstalk}

\section{Comportamiento de las cuentas oscuras}

En la Física experimental de cualquier ámbito, existe una eterna batalla que enfrenta a la señal física buscada con el ruido electrónico y los fenómenos estocásticos que la empañan y esconden en nuestros detectores. Por ello, es crucial adquirir un buen conocimiento de los dispositivos que se utilizan para recoger la señal. Comprender bien su naturaleza interna par$\operatorname{ticular}^{14}$ y su respuesta al resto del equipamiento en el laboratorio se torna un paso clave exactamente igual de importante que el conocimiento de sus magnitudes interesantes como la ganancia estudiada anteriormente.

Con el fin de aportar un marco de resultados experimentales que caractericen los niveles típicos de ruido del SiPM que se encuentra en el laboratorio de la Universidad, en este Trabajo de Fin de Máster vamos a estudiar el ratio de cuentas oscuras, señales espúreas de origen térmico que replican la seña de identidad de la señal física. Adicionalmente, vamos a hacer un estudio de la probabilidad de Crosstalk entre celdas, fenómeno que empañará nuestro conocimiento sobre la llegada de más de un fotón de forma simultánea a varios píxeles.

Como se ha visto anteriormente en la parte II, ahora apagamos todos los instrumentos que no sean necesarios para la monitorización del SiPM. No iluminamos con el LED y, por tanto, el generador de funciones permanece también desconectado. El módulo de la QDC pierde su importancia ahora, que recae sobre el Scaler que se va a encargar de contar las señales que le llegan en función del voltaje umbral dinámico. Ahora, lo único que nos interesa es medir si se generan y registran señales en el SiPM, que seguimos sometiendo a un cierto $V_{b i a s}>V_{b r}$ para que se mantenga en modo Geiger. Idealmente, esto no debiera ocurrir. El SiPM no está iluminado, de tal modo que nadie debería poder generar un par $e-h$ y ninguna avalancha sucedería, dejando la la señal estática en su ruido electrónico habitual.

Pero desgraciadamente no es ideal, el SiPM presenta un ruido que podemos ver en la figura 17 para una $T=-10^{\circ} \mathrm{C}$ y diversos valores del Over Voltage. Incluso si no iluminamos el SiPM y nos situamos en la temperatura más baja que hemos estudiado en este trabajo (es decir, menor agitación térmica y reducción de la probabilidad de sucesos espúreos), el detector nos

\footnotetext{
${ }^{14} \mathrm{Ni}$ siquiera todos los detectores del mismo tipo presentan los mismos niveles de ruido. De ahí que haya que poner a prueba y caracterizar cada detector en un experimento.
} 
muestra claramente que posee una señal. Él está midiendo algo y la electrónica lo confirma.

Primeramente, antes de estudiar la peculiar forma a modo de "escalera" que poseen los datos en la figura 17, podemos ya darnos cuenta de algo que era de esperar. Si la temperatura se mantiene constante, aumentar el $V_{\text {over }}$ (aumentando el $V_{\text {bias }}$ que suministramos al SiPM mediante las fuentes de tensión) incrementa el campo eléctrico interno, de modo que más sucesos de generación espontánea $e-h$ pueden ser registrados acelerando los portadores y multiplicándolos, de tal forma que el ratio de cuentas que superan el umbral dinámico es superior para cualquier valor del voltaje umbral cuanto mayor sea $V_{\text {over }}$.

Es ya momento de analizar la forma que poseen las gráficas de estos resultados en la figura 17. Su comprensión es inmediata mediante la comparación con la fígura 5 y la recuperación de la explicación que se ha dado para esta parte del experimento en la parte II.

Cuando el voltaje umbral, que separa el número de cuentas que mandamos al Scaler de las que son rechazadas, está en torno a $0 \mathrm{mV}$, automáticamente estamos admitiendo absolutamente todas las señales que se produzcan en el detector, puesto que cualquier suceso va a generar una señal por encima de $0 \mathrm{mV}$, señal y ruído van incluidas y no hay forma de separarlas. Esto directamente nos explica el comienzo de la toma de datos en un máximo (nótese la escala logarítmica del eje de ordenadas para hacerse una idea del número de cuentas totales registradas en el máximo respecto a las medidas cuando vayamos aumentando el voltaje umbral).

Un simple vistazo nos lleva a preguntanos la razón por la cual no empieza en $0 \mathrm{mV}$ la toma de datos, sino que se encuentra desplazada algo más de $20 \mathrm{mV}$. La señal procedente del SiPM no es llevada directamente al módulo LTD o al Scaler, sino que se hace pasar previamente por el módulo Fan In / Fan Out (ver Apéndice A), donde se invierte la señal y se le introduce un cierto offset negativo, para tener la señal bien dentro del campo negativo y que los módulos electrónicos puedan procesarla claramente. Así, no es hasta que el voltaje umbral dinámico llega a esas regiones inferiores a $0 \mathrm{mV}$ marcadas por el offset que comienza a ver algo. En otras palabras, nuestro nivel de 0 está desplazado a conciencia en la calibración de las señales para poner de acuerdo las ventanas de trabajo entre los módulos y facilitar su labor.

Con la evolución del voltaje umbral (desplazamiento hacia abajo en la fígura 5), llegamos a un plateau. Ahora que hemos dejado atrás el ruído electrónico estocástico en torno a nuestro 0, superan el umbral todos los picos señal para uno, dos, o varios fotones detectados que dan lugar a una avalancha, razón por la que aquí el ratio de cuentas tomadas por el Scaler es mayor que para mayores umbrales. Durante una región que abarca todo el primer plateau, el voltaje recorre la altura típica de los picos correspondientes a un fotoelectrón (que incluye a los picos de mayor altura relativos a dos o más sucesos simultáneos de detección de fotones). Estos picos de un fotoelectrón son más pequeños que los referentes a la medida simultánea de más fotones, pues la carga recogida es menor, de tal modo que una vez que el voltaje 
umbral sigue aumentando, eventualmente los dejaremos atrás y tan solo estaremos tomando como cuentas válidas aquellas que los superen, es decir, eventos de dos o más fotoelectrones generados simultáneamente en varias celdas. El plateau termina y se produce una caída al tiempo que dejamos de tolerar como válidos los picos de un fotelectrón.

¿Por qué la caída no es abrupta si la altura de los picos en la figura 5 hace referencia solamente a la carga recogida? Sería de esperar que, una vez superamos el nivel de un fotoelectrón, ya sólo midamos las cuentas de dos o más.

Nuevamente, volvemos a la no idealidad. Generalmente, dos sucesos de un fotoelectrón no van a producir exactamente la misma avalancha. Por ello, más que una altura típica o nivel exacto de un fotoelectrón, tenemos una distribución de tamaños típicos para los picos.

De forma sucesiva, el incremento del voltaje umbral va rechazando cada vez más sucesos observándose la esperada sucesión de plateaus y caídas suaves a medida que vamos dejando fuera los niveles de dos, tres, o más fotoelectrones.

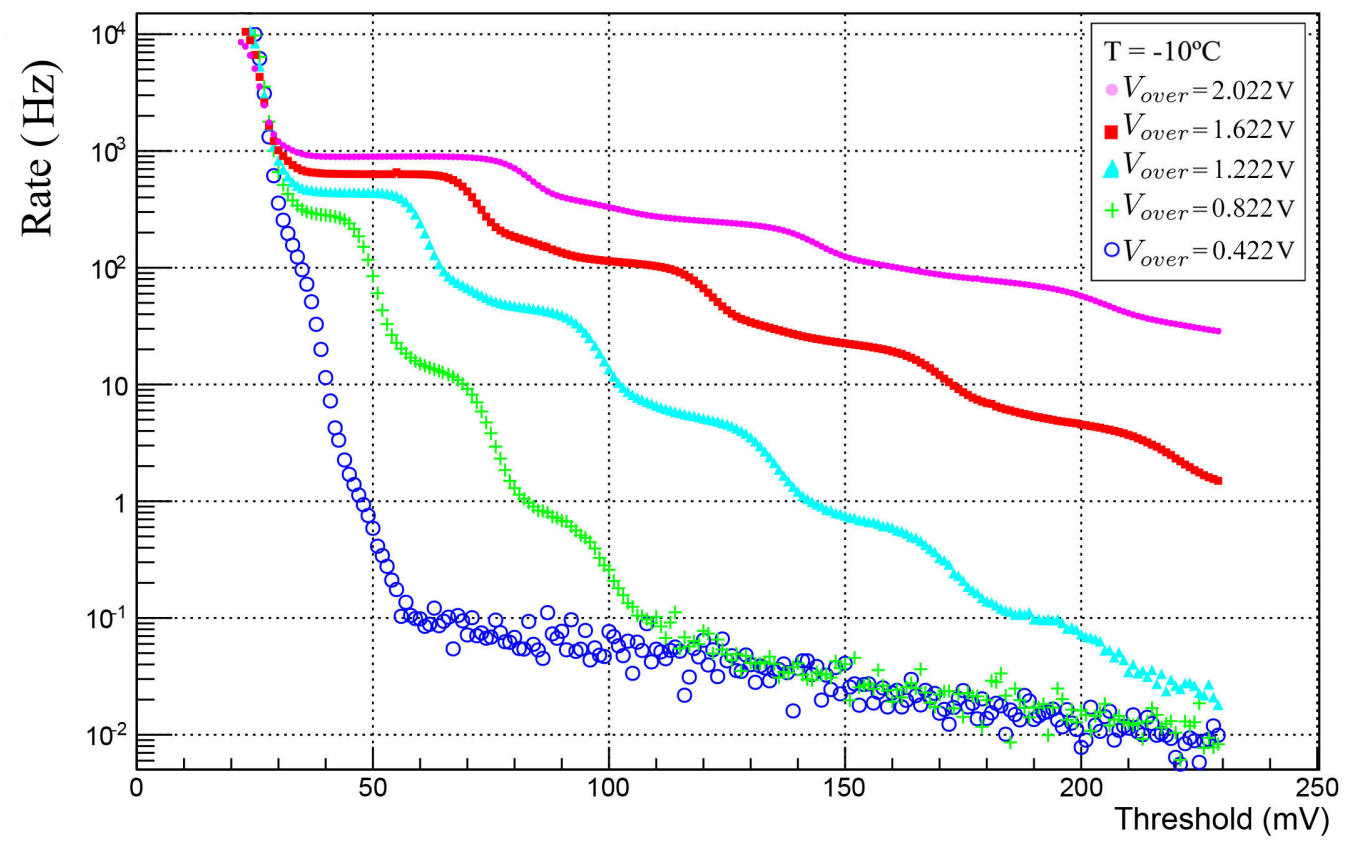

Figura 17: Resultados experimentales para las cuentas oscuras registradas por el SiPM (en ordenadas y en escala logarítmica) en función del voltaje umbral a una $T=-10^{\circ} C$ y para diferentes $V_{\text {over }}$. Los detalles sobre los marcadores y el código de colores utilizado se adjuntan en la leyenda de la esquina superior derecha.

De forma análoga, si antes representábamos las cuentas oscuras que han pasado el veto del voltaje umbral y han sido registradas por el Scaler para una misma temperatura y variando el 
$V_{\text {over }}$, podemos ahora hacer lo contrario: representar los runs para valores similares del Over Voltage, dejando variar la temperatura (figura 18). Como se ha explicado en la parte II, la toma de datos en esta parte se realiza para incrementos de $V_{\text {bias }}$ de $0,4 \mathrm{~V}$ para adecuarnos al mayor tiempo requerido en la toma de datos de cada punto, de modo que no necesariamente vamos a tener runs de medidas para el mismo $V_{\text {over }}$ y para cada temperatura. No obstante, sí son valores muy cercanos cuya diferencia no enmascara en absoluto el resultado físico interesante.

En la figura 18, vemos los resultados que, de nuevo, eran esperables. Para un $V_{\text {over }}$ aproximadamente constante, un incremento de temperatura conlleva una mayor agitación térmica, que produce un aumento en la generación estocástica de pares $e-h \mathrm{y}$, con él, un mayor registro de sucesos que producen avalancha a todos los niveles de voltaje umbral.

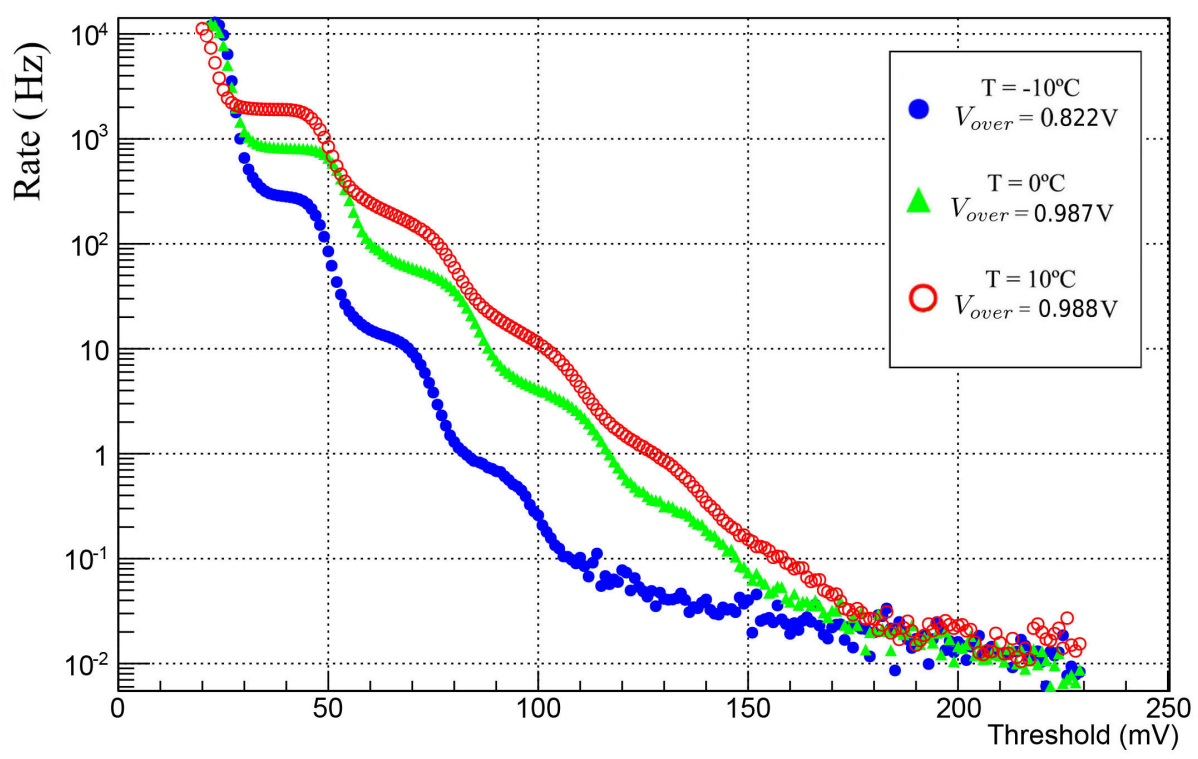

Figura 18: Resultados experimentales para las cuentas oscuras registradas por el SiPM (en ordenadas y en escala logarítmica) en función del voltaje umbral. Se muestran con el código de marcadores y colores de la leyenda de la esquina superior derecha los datos para las temperaturas a un $V_{\text {over }}$ específico para cada una.

Finalmente, en la figura 19, se muestra el Rate de cuentas oscuras en función de $V_{\text {over }}$ para el nivel de 0,5 fotoelectrones y para $T=-10^{\circ} C$. Como se explicará en detalle en la sección 5, el nivel de 0,5 fotoelectrones es aquel que nos asegura que todo lo registrado a voltajes inferiores corresponde a ruido electrónico, y todo lo registrado para umbrales de voltaje superiores corresponde a sucesos espúreos que son de interés en este caso. En la figura 19, podemos observar claramente dicho incremento en la agitación térmica y en la creación de pares que son detectados a medida que el voltaje de operación del SiPM aumenta pues, por 
ejemplo, para un $V_{\text {over }}=0,822 \mathrm{~V}$, el Rate se encuentra en $293 \mathrm{~Hz}$ y, si aumentamos el Over Voltage a $V_{\text {over }}=2,022 \mathrm{~V}$, el Rate se incrementa hasta los $895 \mathrm{~Hz}$.

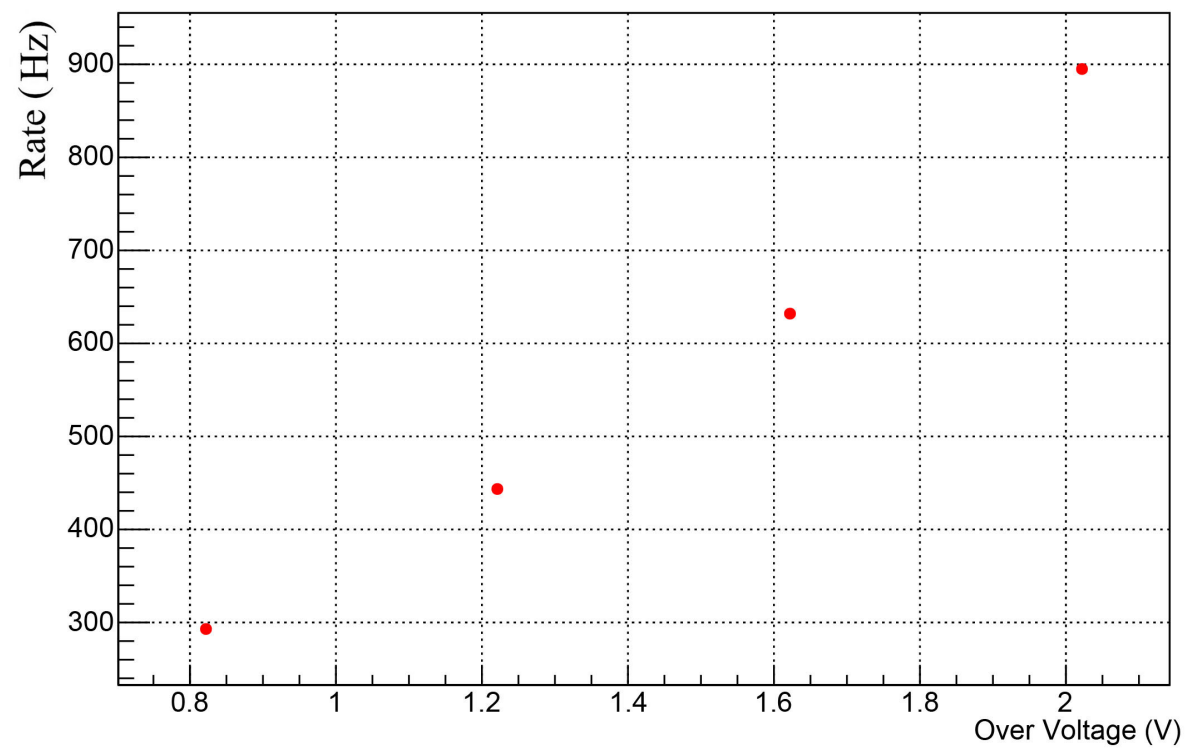

Figura 19: Rate de cuentas oscuras en función del Over Voltage para un umbral superior al nivel de 0,5 fotoelectrones y para $T=-10^{\circ} \mathrm{C}$.

\section{Estimación y resultados de la probabilidad de Cross- talk}

Como vimos en la sección 1.6.2, existe una probabilidad no nula de que los electrones que toman parte en la avalancha de una celda del SiPM emitan un fotón secundario, preferentemente en el infrarrojo, que puede atravesar el detector y accionar una avalancha en otra celda diferente mediante la generación de un par $e-h$ espúreo, suceso que sería indistinguible de la medida simultánea de dos fotones de la señal física de interés en dos celdas diferentes. Se convierte así en otro factor que debemos caracterizar cuidadosamente para tenerlo en cuenta a la hora de utilizar un SiPM en cualquier experimento de Física de partículas. 
Todavía nos queda un comentario fundamental por hacer sobre las gráficas de la sección anterior estudiando las cuentas oscuras que, a conciencia, no se ha puesto de manifiesto todavía. La generación espontánea de pares $e-h$ debido a la agitación térmica de las partículas que forman el detector es un suceso estocástico, de modo que uno podría preguntarse, en relación a gráficas como las de las figuras 17 y 18: ¿Qué significa ver sucesos de dos o más avalanchas registradas en diferentes celdas? ¿Cuál es la probabilidad de que, siguiendo un proceso eminentemente estocástico, se generen espontáneamente pares $e-h$ en varias celdas de forma simultánea? La respuesta es lógica: La probabilidad es pequeña. Se producen sucesos de generación térmica espontánea simultánea, pero no a los niveles registrados en el experimento ya que son sucesos aislados tanto menos probables cuantas más celdas registren una avalancha simultánea sin un emisor externo. Así, lo que estamos viendo en las figuras 17 y 18 es una evidencia clara de que el detector sufre de fenómenos de Crosstalk, en los que sucesivas avalanchas emiten fotones secundarios que activan otras celdas, sucesos mucho más probables que la generación espontánea y aleatoria que sucede de forma simultánea en varias celdas.

Centrémonos ahora en la figura 20 para explicar cómo vamos a inferir la probabilidad de Crosstalk del SiPM del laboratorio. Lo primero que nos preguntamos es cómo podemos estar seguros de que determinamos bien los umbrales de uno, dos o más fotoelectrones. El procedimiento se explica a continuación.

La primera caída que vemos en la figura 20 marca los valores del umbral para los cuales los picos no ideales de un fotoelectrón comienzan a ser rechazados. Debido a que, como se ha mencionado antes, no tenemos una altura característica en los picos sino una distribución de las mismas, la mejor manera de estimar el nivel medio de un fotoelectrón es tomar el valor medio entre todas las alturas que se registran para ellos, lo cual se traduce en tomar el punto medio de la primera transición entre plateaus (linea sólida roja a la izquierda en la figura 20). El valor del threshold para ese punto medio es el valor en $m V$ que toman en promedio los picos de un fotoelectrón.

El mismo procedimiento se va a seguir para estimar la altura media de los picos correspondientes a dos fotoelectrones, tomando el punto medio en la transición entre el segundo y el tercer plateau de la gráfica (lo cual, de nuevo, se traduce en determinar una altura media de los picos referentes a la medida simultánea de dos fotoelectrones). 


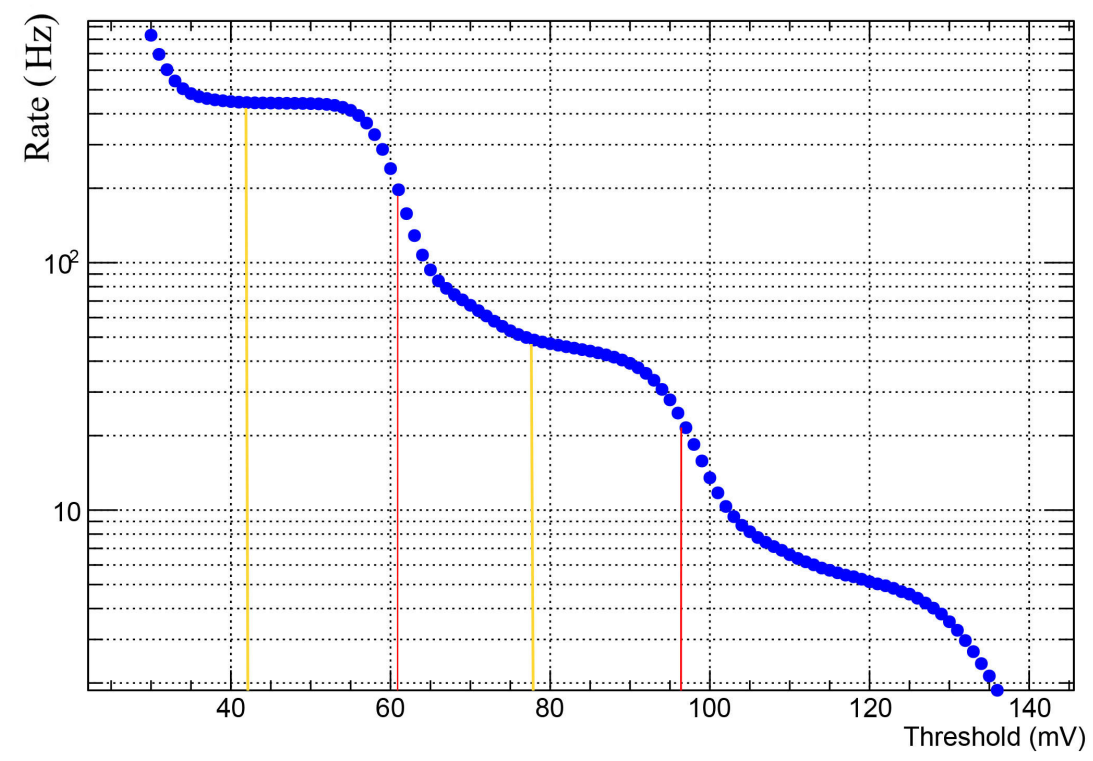

Figura 20: Detalle de las cuentas registradas con el Scaler para un ejemplo concreto a $T=$ $-10^{\circ} \mathrm{C}$ y $V_{\text {over }}=1,2 \mathrm{~V}$. El eje horizontal abarca los tres primeros plateaus del run. Los puntos sólidos azules representan los datos experimentales del laboratorio. Las líneas sólidas rojas marcan los niveles promedio de 1 y 2 fotoelectrones. Las líneas sólidas amarillas marcan los niveles promedio de 0,5 y 1,5 fotoelectrones.

Estimar la probabilidad de Crosstalk equivale a realizar el cociente entre las cuentas registradas referentes a sucesos de dos o más fotoelectrones simultáneos (en su gran mayoría, sucesos producidos por comunicación espúrea entre celdas) y las cuentas totales registradas.

Para asegurarnos de no dejar fuera ningún dato, el cociente que vamos a realizar no será éste exactamente, sino que vamos a utilizar el valor promedio de un fotoelectrón para, dividiendo a la mitad, conocer el valor promedio de 0,5 fotoelectrones. Si realizamos el cociente entre las cuentas registradas por encima del umbral de 1,5 fotoelectrones, $N_{1,5}$, y las cuentas totales registradas por encima de 0,5 fotoelectrones, $N_{0,5}$, el cociente

$$
P_{\text {Crosstalk }}=N_{1,5} / N_{0,5}
$$

nos asegura que, aprovechando la altura considerable de los picos, estamos realmente incluyendo todos los eventos por encima de un fotoelectrón en el numerador, y todos los eventos debidos a uno, dos, o más fotoelectrones en el denominador, sin ningún tipo de pérdida de generalidad o incertidumbre relevante asociada a la estimación de los puntos medios de las transiciones entre plateaus. 

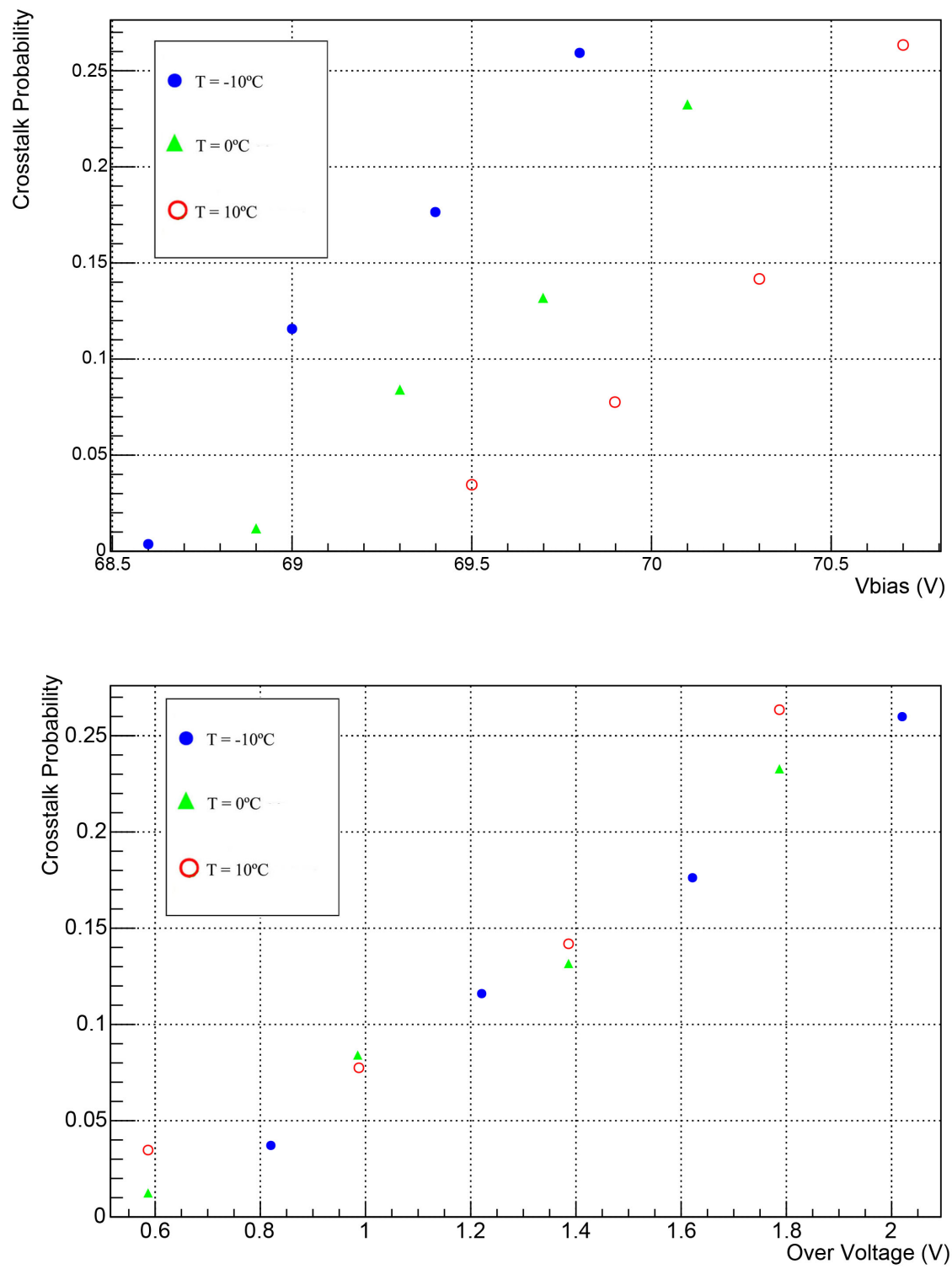

Figura 21: Resultados experimentales para la dependencia de la probabilidad de que sucedan fenómenos de Crosstalk con el $V_{\text {bias }}$ (arriba), y con el $V_{\text {over }}$ (abajo), para todas las temperaturas analizadas en el laboratorio. Los detalles sobre los marcadores y el código de colores utilizados se adjuntan en las leyendas de la esquina superior izquierda.

Con este método, utilizamos todas las tomas de datos para las tres temperaturas medidas en el laboratorio, $T=-10,0,10^{\circ} \mathrm{C}$, y determinamos los puntos que dan lugar a la dependencia de la probabilidad de Crosstalk con el voltaje de bias en la gráfica superior de la figura 21, y con el Over Voltage en la misma figura pero en la gráfica inferior.

Comencemos el análisis de los resultados por la gráfica superior en la figura 21. Lógicamente, para valores crecientes del voltaje de bias la probabilidad de Crosstalk aumenta ya que aumenta 
el número de portadores involucrados y acelerados por el campo eléctrico en la avalancha y por tanto existe una mayor probabilidad de que alguno de ellos emita un fotón que sea registrado por otra celda. El comportamiento creciente es el esperado. Por otra parte, sabemos que el régimen de operación en modo Geiger del SiPM se desplaza a valores de $V_{\text {bias }}$ altos cuando aumentamos la temperatura (ver la figura 15) lo cual explica sin lugar a dudas por qué las curvas de la probabilidad de Crosstalk se desplazan hacia la derecha al aumentar la temperatura.

Nuevamente, de forma similar a lo ocurrido en la figura 16, si lo que hacemos ahora es representar esta misma probabilidad de Crosstalk pero frente al $V_{\text {over }}$, dejamos de tener en cuenta los efectos de la temperatura sobre el régimen de operación del detector y comprobamos que hay un colapso de todas las curvas a una sola, dando buena cuenta de que la probabilidad de Crosstalk es un parámetro intrínseco característico del detector. Las temperaturas altas no aumentan la probabilidad de Crosstalk, lo que hace que observemos más comunicación entre celdas es aumentar el voltaje de operación, ya que eso hará intervenir más portadores que pueden radiar.

Para finalizar este apartado, cabe realizar un comentario sobre cómo puede reducirse la probabilidad de que se produzcan fenómenos de Crosstalk. Operar con el SiPM en un régimen de baja ganancia reduce dicha probabilidad mediante la reducción del número de portadores por avalancha, pero a costa de reducir significativamente la eficiencia en la detección de fotones, ya que se complica la aceleración del fotoelectrón primario. Una alternativa conveniente para reducir el Crosstalk es insertar ranuras entre las celdas que estén llenas de un absorbente óptico. Esto, sin embargo, no viene carente de defectos, pues el espacio que requiere su inserción reduce el área efectiva del detector, reduciendo con ella el factor de llenado que se describía en la subsección 1.3 e indirectamente reduciendo también la PDE. La opción preferente es alcanzar un equilibrio entre todos estos factores y monitorizar la probabilidad de Crosstalk para conocerla bien y tenerla en cuenta en los experimentos a realizar con el SiPM, como se ha hecho en este Trabajo. 



\section{Parte V}

\section{Conclusiones}

Los fotomultiplicadores de silicio han revolucionado el campo de los detectores de fotones por su elevada eficiencia y ganancia, bajos niveles de ruido, buena respuesta y compactas dimensiones.

Gracias al desarrollo de este Trabajo de Fin de Máster, se ha podido llevar a cabo la caracterización del SiPM del laboratorio de la Universidad de Granada. Conocemos la respuesta del detector ante los impulsos externos de una fuente luminosa como un LED, sabemos cómo se comporta cuando uno de los fotones incidentes genera pares de portadores que son acelerados, creando una cascada en la que se amplifica su número de acuerdo a una ganancia intrínseca del detector, que hemos medido experimentalmente para diversos intervalos del voltaje de alimentación y para diversas temperaturas. Así, hemos verificado experimentalmente la dependencia lineal de la ganancia con el $V_{\text {bias }}$ para diversas temperaturas, y la linealidad del voltaje de entrada a modo Geiger, el Breakdown Voltage, con dicha temperatura.

Gracias a esto, el SiPM nos permite detectar señales tan débiles como las generadas por un único fotón con gran precisión. Adicionalmente, se han podido determinar los intervalos de voltaje para los cuales el SiPM trabaja en modo Geiger de forma clara, una vez rebasado el Breakdown Voltage. Gracias a este análisis, se establece una base que propicia la buena bondad de las medidas que se deseen tomar en experimentos futuros en Física de partículas, Astrofísica u otras áreas del conocimiento que precisarán de la utilización del SiPM que hemos caracterizado.

La continua batalla señal-ruido en el análisis de datos requiere un profundo conocimiento de la estructura interna del detector y de su tendencia a empañar los sucesos físicos con señales espúreas que, junto al ruído electrónico, pueden llegar a impedir la correcta interpretación de los resultados arrojados por un experimento. Por ello, se ha dedicado en este Trabajo una importante parte del tiempo al estudio del ruido del SiPM a modo de generación térmica espontánea de portadores y de comunicación entre celdas. Hemos medido las cuentas oscuras y el Crosstalk del detector para varias temperaturas y voltajes de bias, cuantificando el ruido. De esta manera, hemos aportado una parametrización de los efectos espúreos del SiPM del laboratorio, que pueden ahora compararse con otros modelos de fotomultiplicadores de estado sólido del mercado y que pueden aprovecharse para interpretar correctamente futuros experimentos con el detector de este laboratorio.

Una importante parte de este Trabajo de Fin de Máster ha sido la introducción al trabajo en un laboratorio de Física de Partículas, teniendo la oportunidad única de aprender a 
desenvolverse en un laboratorio especializado, a solventar los problemas típicos que en ellos suelen surgir (comprender por qué una señal no se comporta como debiera, entender la importancia del orden y la rigurosidad que un laboratorio requiere, modificar la instrumentación para obtener nuevas señales que aporten información complementaria, etc.), a comprender la instrumentación electrónica y sus canales de comunicación con el detector, a trabajar con la electrónica NIM y VME, entre otras muchas cosas.

El desplazamiento a un laboratorio y el trabajo en el mismo son tareas que requieren de alta disciplina, de métodos eficientes de trabajo que permitan obtener buenos resultados al tiempo que cuidan la instrumentación. Estas tareas y métodos son esbozados de forma escasa durante la carrera y el Máster, en los cuales la enseñanza es eminentemente teórica y la base de laboratorio (y más en uno especializado en Física de partículas) es débil, de tal modo que la realización de este Trabajo de Fin de Máster ha sido un paso importante de mi formación, culminando una etapa mediante la aportación de un profundo conocimiento complementario y sentando una importante y versátil base para la siguiente. 


\section{Referencias}

[1] RCA Corporation, "RCA Photomultiplier Manual", PT-61 (1970).

[2] Tsang, W.T., "Semiconductors and Semimetals", Vol. 22, Part D "Photodetectors". Academic Press (1985).

[3] ROOT. Data Analysis Framework. https://root.cern.ch/drupal/

[4] D. Renker y E. Lorenz, "Advances in solid state photon detectors", 2009 JINST 4 P04004.

[5] Claudio Piemonte et al., "Characterization of the First Prototypes of Silicon Photomultiplier Fabricated at ITC-irst", IEEE Nuclear Science, Vol. 54, n. 1, Feb. 2007.

[6] K. Deiters et al., "Investigation of the avalanche photodiodes for the CMS electromagnetic calorimeter operated at high gain", Nucl. Instrum. Meth. A 461 (2001) 574.

[7] B. Lewandowski, "A fast and compact electromagnetic calorimeter for the PANDA detector at GSI", PANDA Collaboration, Nucl. Instrum. Meth. A 537 (2005) 349.

[8] M. Moszynski et al., "Large area avalanche photodiodes in scintillation and X-rays detection", Nucl. Instrum. Meth. A 485 (2002) 504.

[9] R. Lecomte et al., "Initial results from the Sherbrooke avalanche photodiode positron tomograph", IEEE Trans. Nucl. Sci. 43 (1996) 1952.

[10] C. Woody et al., "RatCAP: a small, head-mounted PET tomograph for imaging the brain of an awake RAT", Nucl. Instrum. Meth. A 527 (2004) 166.

[11] B. Pichler et al., "Positron emission tomography/magnetic resonance imaging: the next generation of multimodality imaging?", Semin. Nucl. Med. 38 (2008) 199.

[12] A. Braem et al., "Wavelength shifter strips and G-APD arrays for the read-out of the z-coordinate in axial PET modules", Nucl. Instrum. Meth. A 586 (2008) 300.

[13] Y. Kudenko, "The near neutrino detector for the T2K experiment", Nucl. Instrum. Meth. A 598 (2009) 289.

[14] Maurice Stephan et al., "Future use of silicon photomultipliers for the fluorescence detection of ultra-high-energy cosmic rays", Proc. SPIE 8155, Infrared Sensors, Devices, and Applications; and Single Photon Imaging II, 81551B (September 16, 2011); doi:10.1117/12.893089. 
[15] A. Stoykov et al., "First experience with G-APDs in m SR instrumentation", NDIP08, to be published in Nucl. Instrum. Meth. A.

[16] CAEN. Tools for Discovery. http://www.caen.it/

[17] Hamamatsu Photonics K.K., Solid State Division, "Hamamatsu SiPM book".

[18] A. N. Otte et al., "Status of Silicon Photomultiplier Developments as optical Sensors for MAGIC/EUSO-like Detectors", 29th International Cosmic Ray Conference Pune (2005)00, 101-106.

[19] Valeri Saveliev (2010). Silicon Photomultiplier - New Era of Photon Detection, Advances in Optical and Photonic Devices, Ki Young Kim (Ed.), ISBN: 978-953-7619-76-3, InTech

[20] Patrick Eckert et al., "Characterisation Studies of Silicon Photomultipliers", arXiv:1003.6071v2 [physics.ins-det] 1 Apr 2010.

[21] Sense light Technical Note, "An Introduction to the Silicon Photomultiplier", Rev. 3.1, October 2011, www.sensl.com 


\section{A. Descripción de los módulos electrónicos.}

En este Apéndice, se va a dar una breve descripción de la instrumentación electrónica utilizada para tratar las señales eléctricas en el experimento y que están disponibles en el laboratorio de la Universidad de Granada. Los módulos han sido desarrollados y construídos por una de las empresas líderes mundiales en instrumentación que proveen a los mejores laboratorios, centros de investigación y universidades del mundo, CAEN (Costruzioni Apparecchiature Elettroniche Nucleari S.p.A., [16]).

De izquierda a derecha, los módulos utilizados en las capturas de la Figura 22 son:

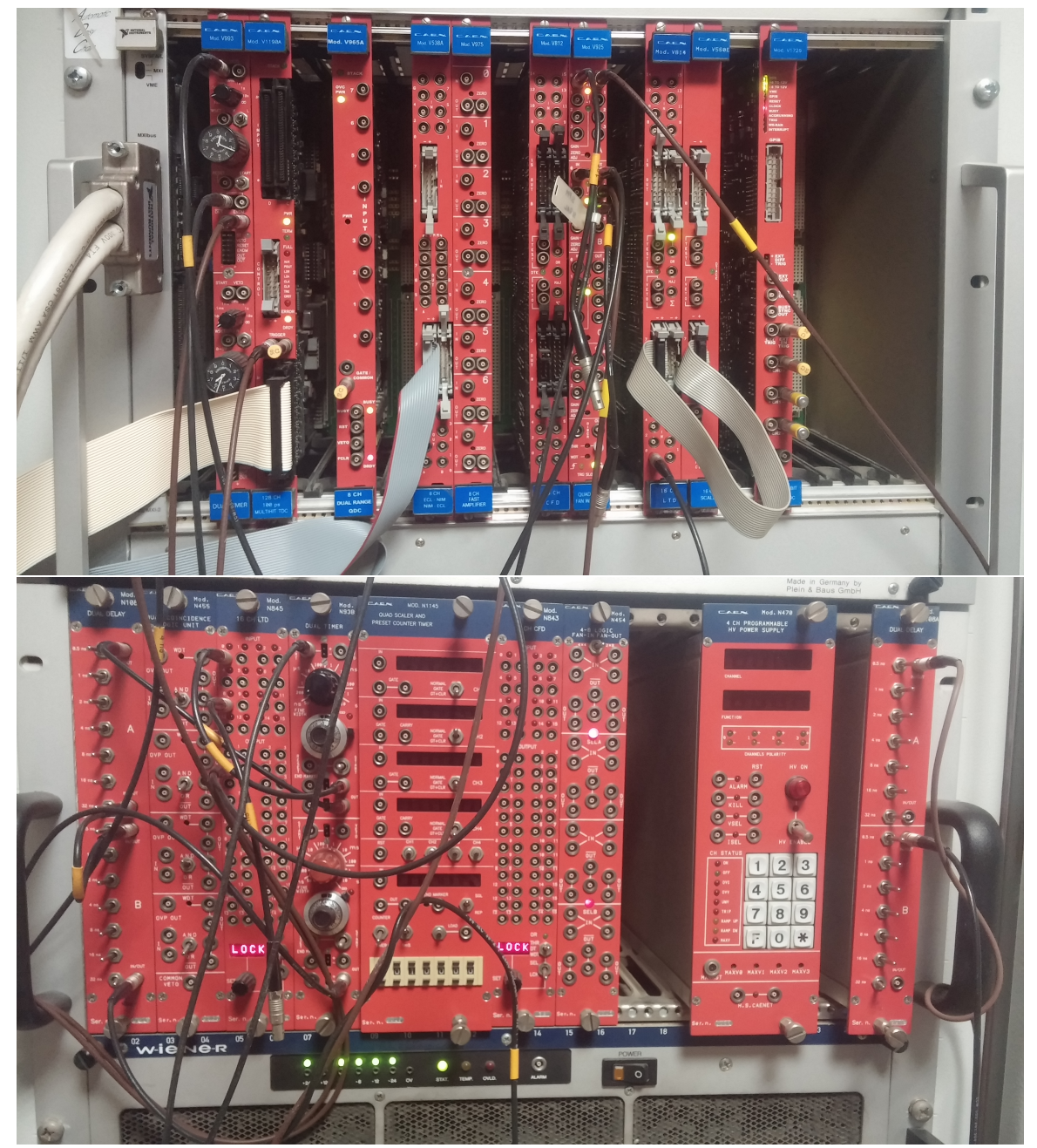

Figura 22: Detalle de los módulos para el tratamiento de la señal del SiPM que se encuentran en el Rack visible en la parte derecha de la primera imagen en la figura 6.

\section{Dual Timer}

El módulo V993 Dual Timer es un módulo VME $^{15}$ que aloja dos generadores de pulsos con el

${ }^{15}$ El VMEbus es un estándar de bus informático. 
mismo trigger. El módulo produce pulsos NIM/TTL y ECL ${ }^{16}$ cuyas anchuras varían entre 50 ns y 10 ns cuando son activados. Los pulsos de salida son entregados normal y negado, respectivamente.

El trigger de los timers puede ser recalibrado, al igual que la anchura de salida, vía el switch rotatorio de nueve posiciones del frontal o aportando un voltaje externo. El START del trigger puede ser controlado por una vía externa (NIM, TTL o ECL), o manualmente vía un switch en el panel frontal. El módulo también realiza vetos y reinicios de las señales de entrada. Además, cuenta con un tiempo de subida/bajada de la señal inferior a 2 ns.

\section{QDC}

El módulo V965A consta de ocho canales conversores de "carga-a-digital" de impedancia $50 \Omega$, polaridad negativa, acoplamiento DC y con una resolución de 12 bits (15 bits dinámicos). Para cada canal, la carga entrante es convertida a un nivel de voltaje mediante la QAC (conversión de carga a amplitud). Cada salida QAC es convertida por dos ADC's (conversor análogico-digital) en paralelo a una señal digital legible por el correspondiente código de ordenador. La señal de entrada a la QDC puede leerse en dos escalas gracias al Dual Range: $0 \div 900 p C / 0 \div 100 p C$, que permite obtener una amplia ventana de integración con menor resolución o una ventana de integración más estrecha con una resolución mejorada.

Sus controles de entrada NIM son:

- GATE: ventana temporal de integración que debe preceder a la entrada analógica por más de 15 ns.

- RST: reinicia las secciones QAC y los registros de control.

- VETO: suprime la conversión de señales QAC.

- FCLR: limpieza rápida de las secciones QAC.

Sus controles de salida NIM son:

- DRDY: indica la presencia de datos.

- BUSY: placa llena, reiniciando, convirtiendo o en modo de verificación de memoria.

La supresión programable, buffer de memoria multi-evento, contador de triggers y herramientas de testeo completan la flexibilidad de esta unidad.

\footnotetext{
${ }^{16}$ Los Módulos de Instrumentación Nuclear (Nuclear Instrumentation Module, NIM) estándar definen especificaciones eléctricas y mecánicas para los módulos electrónicos utilizados en física de partículas experimental y física nuclear. Así, se potencia la flexibilidad, el intercambio de instrumentos, el esfuerzo de diseño, el mantenimiento y la mejora de los mismos. Por su parte, TTL hace referencia a circuitos Transistor-Transistor-Logic que incorporan transistores de unión bipolar, BJT's. ECL es un entorno de programación y desarrollo creado por la Universidad de Harvard en los años 1970.
} 


\section{Fan-In / Fan-Out}

El módulo V925 es un VME que alberga tres secciones 4 In / 4 Out y una sección 3 In / 3 Out, además de un canal discriminador. Cada sección del Fan In / Fan Out produce para todos sus conectores de salida la suma de todas las señales de entrada invertidas. Las entradas son bipolares mientras que las salidas pueden invertirse o permanecer intactas (ajustable de forma independiente para cada sección), estando ambas acopladas directamente por un medio conductor ( $D C$ coupling). La máxima amplitud de entrada es $\pm 1,6 \mathrm{~V}$. Además, cada sección permite ajustar un offset de forma independiente.

Los LED's del panel frontal permiten monitorizar todos los modos, la ganancia y la polaridad de los puentes internos.

\section{Low Threshold Discriminator (LTD)}

El discriminador de bajo umbral V814 es un módulo VME que alberga 16 canales discriminadores. El módulo acepta 16 entradas negativas o positivas y produce 16 salidas ECL diferenciales. La frecuencia máxima de entrada son $60 \mathrm{MHz}$.

El discriminador produce una pulso de salida con una anchura ajustable entre 6 ns y 95 ns vía VME. Los umbrales son ajustables a través del ordenador en el intervalo que abarca desde $-1 m V$ hasta $-255 \mathrm{mV}$ (con pasos de $1 \mathrm{mV}$ ). También existe una versión, con inputs positivos, y con umbrales desde $1 \mathrm{mV}$ hasta $255 \mathrm{mV}$. Adicionalmente, cada canal puede encenderse o apagarse de forma independiente.

\section{Scaler}

El módulo V560E alberga 16 canales independientes de contaje de 32 bits con una frecuencia máxima de entrada de $100 \mathrm{MHz}$. Cada canal puede ser habilitado vía software para generar una señal que se interrumpe cuando el contador ha llegado a un máximo de cuentas ajustable. Cada canal puede enlazarse en cascada con el siguiente via puentes internos para producir una profundidad de contaje de hasta 64 bits. El estado de cada uno de los puentes internos puede ser leído vía ciclos de lectura estándar VME.

Todos los canales pueden ser reiniciados mediante el comando VME correspondiente o con el botón frontal del panel.

\section{Dual Delay}

El módulo N108A es una unidad de desfase dual en un único módulo NIM. El desfase varía en el intervalo que engloba desde 0 ns a 63,5 ns (+1,6ns de offset) por sección. El desfase se puede fijar en pasos de $0,5 \mathrm{~ns}$ y se consigue con cables coaxiales calibrados que no requieren una fuente de alimentación externa. 